\title{
Coalition Formation as a Dynamic Process*
}

\author{
Hideo Konishi \\ Department of Economics, Boston College, Chestnut Hill, MA 02467, USA \\ E-mail: hideo.konishi@bc.edu \\ and \\ Debraj Ray \\ Department of Economics, New York University, 269 Mercer Street, New York, NY \\ 10003, USA, and Instituto de Análisis Económico (CSIC) \\ E-mail: debraj.ray@nyu.edu

\begin{abstract}
We study coalition formation as an ongoing, dynamic process, with payoffs generated as coalitions form, disintegrate, or regroup. A process of coalition formation $(\mathrm{PCF})$ is an equilibrium if a coalitional move to some other state can be "justified" by the expectation of higher future value, compared to inaction. This future value, in turn, is endogenous: it depends on coalitional movements at each node. We study existence of equilibrium PCFs. We connect deterministic equilibrium PCFs with unique absorbing state to the core, and equilibrium PCFs with multiple absorbing states to the largest consistent set. In addition, we study cyclical as well as stochastic equilibrium PCFs. Journal of Economic Literature Classification Numbers: C71, C73.
\end{abstract}

Key Words: coalition formation, farsightedness, dynamic equilibrium

\footnotetext{
* Initial research on this project was conducted when Konishi was a visiting scholar at Boston University, and Ray was a faculty member at Boston University. Konishi thanks KIER at Kyoto University for hospitality and financial support. Ray gratefully acknowledges a fellowship from the John Simon Guggenheim Foundation. We thank Michael Chwe, Bhaskar Dutta, Aki Matsui, Shigeo Muto, Yves Sprumont, Rajiv Vohra, Peyton Young and especially Hsueh-Ling Huynh for helpful comments at various stages of this research. We also thank an associate editor and an anonymous referee whose comments shaped the current version of this paper. This paper is dedicated to the memory of Bob Rosenthal.
} 


\section{INTRODUCTION}

\subsection{A Process of Coalition Formation}

Let $N$ be a set of players and $X$ a set of states. Suppose that for each state in $X$ and each coalition $S$ (a nonempty subset of $N$ ), a possible set of "coalitional moves" (by $S$ ) to some subset of states is given. A map from the current state to a probability distribution over the set of all coalitional moves feasible at that state induces a dynamic process on $X$. Noting that moves are associated with actions taken by coalitions, we call this a process of coalition formation.

Under such a process players receive (additive discounted) utility from the entire path of states. This induces a value for each player in the standard way, as a function of the going state.

A process of coalition formation is an equilibrium if at any date and at any going state, a coalitional move to some other state can be "justified" by the very same scheme applied in future: the coalition that moves must have higher present value (starting from the state it moves to) for each of its members, compared to (one-period) inaction under the going state. In the most general form that we study it, a process of coalition formation precipitates a Markov process on $X$, the uncertainty reflecting both the choice of the deviating coalition at some state (there may be several potential deviants) and the choice of state that the coalition deviates to (there may be several potential moves). At the same time, we do restrict the class of moves by requiring that for each coalition, moves must be Pareto-efficient for members of that coalition, under the value functions induced by the overall process of coalition formation.

The use of value functions induced by the scheme itself implies perfect foresight on the part of all coalitions: players expect and understand that coalitions may move in the future, and form (common) beliefs about the likelihood of such events.

A model of real-time moves demands a proper interpretation of the time period. Our interpretation is that each time period is an interval for which a coalition structure (and the associated actions and payoffs) remains a binding agreement. At the beginning of each new period, a fresh agreement can be written, with the going state a historical (but not legal) status quo.

\subsection{Potential Contribution}

The theory of coalition formation has traditionally belonged to the realm of cooperative game theory (see, for instance, notions of the core, the bargaining set, or the stable set of von Neumann and Morgenstern). Recent literature takes this theory in three important methodological directions. 
First, characteristic functions are dispensed with. ${ }^{1}$ A theory can be developed for situations with widespread externalities, thereby bringing strategic games directly into the picture. Second - and quite unlike notions of the core or the bargaining set - the theory seeks "consistent" formulations, in the sense that considerations of "credibility" are imposed on the blocking coalition in just the same way as they are on the original situation. ${ }^{2}$ Finally, the theory models players as being farsighted, in the sense that they care about the "ultimate" payoff from a move, and not its immediate consequences. ${ }^{3}$

The static version of the framework we use embodies several of the models in the literature. But the explicitly dynamic nature of our definition possesses at least three advantages relative to existing formulations.

First, by allowing all moves to take place in real time, as it were, the definition allows us to bridge the gap between myopic notions of stability (such as those implicit in the core or the bargaining set) and the more recent definitions based on farsightedness (such as those in [2], [6], [11], [23], [27], [28] and [34]) by simply changing the discount factor of agents. Extreme myopia would correspond to a discount factor of zero, while extreme farsightedness would be approximated as the discount factor converges to unity. [It should be added that we are particularly interested in the latter case.]

The point is that the static concepts based on farsightedness are really attempting to capture a fundamentally dynamic process, in which an action may generate a long chain of reactions. In the current paper, we take this dynamic story seriously instead of writing down a shorthand for it.

Second, the theory of blocking and coalitional deviations has been complicated (if not hindered) by the issue of multiple continuations following a single deviation. For instance, Greenberg's approach [17] distinguishes between optimistic and conservative "standards of behavior", in which currently deviating coalitions evaluate the future multiplicity of other deviations in hopeful or pessimistic ways (see also [11], [26], and [34], which all suffer from this selection problem). This Knightian approach to the treatment of multiplicity can be avoided by borrowing more freely from the language of repeated or dynamic games, which we do. Future paths (perhaps probabilistic in nature) are evaluated using common beliefs (as embodied in the transition probability) and expected payoffs are calculated using these beliefs.

\footnotetext{
${ }^{1}$ See, for example, [5], [6], [7], [10], [13], [20], [27], [28] and [35]. An early concern with the limitations imposed by characteristic functions is to be found in [29] and [33].

${ }^{2}$ In contexts where binding agreements can be written, see, for example, [12]. [14], [17] and [26].

${ }^{3}$ See, for instance, [2], [11], [23] and [34].
} 
Third, several solution concepts, especially those that concern themselves with farsighted agents, inevitably run into the problem of cycles (for an early discussion of this, see [31]). Chains of coalitions may appear and reappear in the blocking process. ${ }^{4}$ In the present approach, recurrent cycles of moves pose no problem at all. Payoffs from such cycles are simply to be evaluated as any sequence of payoffs is evaluated: by adding up discounted one-period returns over time.

A particularly relevant interpretation of cyclical outcomes arises from the possibility of constant renegotiation. Agreements may be torn up and rewritten, especially if the environment external to a particular coalition is altered by the formation of other coalitions (note that this would be irrelevant for characteristic functions, but especially important when there are widespread externalities). Is the possibility of such ongoing renegotiation to be ignored, as they will be if cyclical possibilities are somehow closed off?

To be sure, an explicit dynamic model also raises critical questions. Particularly relevant is the assumption of a Markov strategy for all coalitions. The extent of cutting power our model retains if all history-dependent strategies are allowed is an interesting and difficult open question. It should be added, however, that this sort of criticism also applies to the static models of farsightedness, in that the move at some node is taken to be invariant with respect to the mode of arrival at that node.

\subsection{Summary of the Results}

We begin with a formal description of intertemporal coalition formation (Section 2). Our main limiting assumption is that the state space $X$ is finite. The extent to which our results can be extended to infinite state spaces remains an open question. In Theorem 3.1, we show that an equilibrium process of coalition formation exists (we use finiteness of the state space but otherwise the model is perfectly general).

We then proceed to "benchmark" our solution, using familiar concepts from existing literature. To do this, we study the class of deterministic process of coalition formations. We show that in all models of coalition formation that are derived from an underlying characteristic function, the class of deterministic process of coalition formations with unique limit states (essentially) characterizes the core (Theorems 4.1 and 4.2), provided that discount factors are close enough to unity. Apart from benchmarking our solution concept, this result is of independent interest because it reveals

\footnotetext{
${ }^{4}$ One approach is to exclude cycles explicitly by assuming the nestedness of coalitional moves ([4], [14], [26] or [27]). Alternatively, one might exclude cycles by implicitly assuming that such cycles gives the worst payoffs ([23], [34]). Finally, one might study coalition formation in a bargaining context, in which infinite bargaining delays result in zero payoff ([6], [9], [24] and [28]).
} 
an interesting consistency property of the core (which goes beyond the "internal consistency" of the core established in $[26] .{ }^{5}$

Next, we consider deterministic schemes that do not necessarily have a unique limit (but nevertheless do not display cycles). We show by means of an example that non-core limits might now emerge. However, it turns out that such schemes yields absorbing states that always lie within the "largest consistent set" ([11]), provided that discount factors are close enough to unity. This result (Theorem 4.3) is valid without any restrictions on the underlying model of coalition formation.

However, the inclusion result of Theorem 4.3 is generally strict. Example 7, which shows this, brings out the fact that our solution concept imposes more restrictions on the final outcomes than the largest consistent set does. But this does not mean that the outcomes selected by our solution are necessarily the "more efficient" ones; Example 8 is devoted to an understanding of this point.

Next, we make some observations on cyclical solutions. These typically exist in situations in which core-like restrictions lead to an empty outcome. But there are examples in which no cyclical solution (and indeed, no deterministic solution) exists. This motivates a study of probabilistic solutions, which is the final section of the paper.

Uncertainty enters a process of coalition formation in two possible ways. First, a particular coalition may be able to induce two or more states which are not payoff-comparable, and might randomize (or be perceived as randomizing). Second, it is possible that at some state several coalitions have access to profitable moves, and that these are chosen randomly.

It turns out that such forms of randomization occur naturally in strategic form games, in the sense that randomization is often necessary for existence of an equilibrium process (contrast this with characteristic functions). Accordingly, we focus in the section on games in strategic form. The simplest (though by no means trivial) starting point is games with common payoffs. We show that for such games, every equilibrium must lead to the efficient outcome, provided that discount factors are close to unity (Theorem 5.1).

But this result fails when we depart from common payoffs. For instance, we show (Example 11) that a $2 \times 2$ symmetric coordination game may generate equilibria that hone in on the "bad" equilibrium. The stochastic

\footnotetext{
${ }^{5}$ In [16] and [30], it is shown that starting from an arbitrary state, a sequence of profitable coalitional deviations lead to a core state in exchange economies and in TU games, respectively. There is a fundamental difference between these results and the one established here. They assume that players are myopic, so that members of a moving coalition do not foresee what happens after their immediate deviation takes place. In the model described here, individuals are farsighted and will need to forecast future deviations or moves.
} 
nature of the equilibrium is explained in detail; indeed, we argue that such an equilibrium must be stochastic.

We turn finally to a detailed analysis of the Prisoners' Dilemma. Our solution concept applied here yield a rich variety of outcomes (though, to be sure, not everything is possible). The main points are: (1) cooperation can be sustained using deterministic schemes, while defection can never be sustained in this way (provided that discount factors are close to unity); (2) in contrast, stochastic schemes can support defection as an absorbing state, and can also generate cycles of movement with possibly some inertia at the cooperative outcomes; and (3) cardinalities do matter in pinning down equilibria - Example 12, which concludes the paper, makes this amply clear.

\section{COALITION FORMATION}

\subsection{Basic Ingredients}

We consider a dynamic model of coalition formation. Let $N$ be a finite set of players and $X$ a finite set of states. ${ }^{6}$ Using the language of cooperative game theory, one might interpret a state to be the description of a coalition structure, as well as a vector of payoffs accruing to each player. In noncooperative games in strategic form, a state would represent a profile of actions taken in the stage game.

A coalition is a nonempty subset $S$ of $N$. For each state $x$ in $X$ and each coalition $S$, define $F_{S}(x)$ to be the set of states achievable by a one-step coalitional move (by $S$ ) from $x$. A coalition always has the option to do nothing, so we include $x$ in this set.

Let $F(x)$ be the set of all moves from $x$; that is, $F(x)=\cup_{S} F_{S}(x)$.

For each player, there is a vN-M payoff function $u_{i}: X \rightarrow \mathbb{R}$ and a discount factor $\delta_{i}$. Thus player $i$ 's payoff from a sequence of states $\left\{x_{t}\right\}$ may be written as

$$
\sum_{t=0}^{\infty} \delta_{i}^{t} u_{i}\left(x_{t}\right) .
$$

This is easily extended to probabilistic paths. Let $\Delta(X)$ be the space of all probabilities $\sigma$ on $X$. Then for any sequence $\sigma \equiv\left\{\sigma_{t}\right\}$ in $\Delta(X)$, player

\footnotetext{
${ }^{6}$ The restriction to a finite set of states is for technical convenience. At present, we do not know whether results such as Theorem 3.1 extend to the infinite case. For instance, Theorem 3.1 uses a fixed point argument that will need to be extended to spaces of infinite-dimensional transition probabilities.
} 
$i$ 's payoff is given by the expression

$$
U_{i}(\sigma)=\sum_{t=0}^{\infty} \delta^{t}\left(\sum_{x \in X} \sigma_{t}(x) u_{i}(x)\right) .
$$

\subsection{Examples}

Notice that the static, deterministic version of this model embodies several standard models, such as characteristic functions and games in strategic form. But it can also encompass games in partition function form ([27], [29], [33]) or networks ([13], [20]). Our more abstract description has been used by several authors (in its static deterministic version): [11], [17] and [34] represent some recent instances.

As concrete examples, consider the following:

Example 1. A Characteristic Function. Consider the simplest twoperson NTU characteristic function, in which there are simply two coalition structures with a single payoff vector in each. Let $x_{1}$ be the state with structure $\{12\}$, and $x_{2}$ the state with singleton structure. Then for $x \in$ $\left\{x_{1}, x_{2}\right\}, u_{i}$ is just the payoff to player $i$ under the corresponding structure.

It is easy to describe $F_{S}$ as the formalization of what each coalition can do at each state. For example, the singletons can both move at $x_{1}-$ precipitating $x_{2}$ - while the grand coalition of two players can move at $x_{2}$, precipitating $x_{1}$. Whether the grand coalition can move or not at $x_{1}$ to precipitate $x_{2}$ is a matter of interpretation. We are comfortable with either.

Example 2. A Partition Function. Suppose that there are three players. If all three stand together, the payoff vector is $(a, a, a)$. If all stand alone, the payoff vector is $(0,0,0)$. If $i$ is alone and $j k$ are together, the payoff is $b$ to $i$ and $c$ each to the other two. These determine the functions $u_{i}(x)$ for each player $i$ and and each state $x$.

Once again, much of the description of $F_{S}$ is obvious, espcially if we have adopted an interpretative convention as suggested in Example 1. But partition functions pose new issues. Suppose that $i$ moves from the grand coalition. Is the resulting structure $\{i\},\{j k\}$ or $\{i, j, k\}$ ? This time it is more than a mere question of interpretation, and the dynamic model of coalition formation just described forces us to take a stand on the matter. So far as the formal theory is concerned, this is not an issue as long as $F_{S}(x)$ is fully specified for all coalitions $S$ and states $x$.

Example 3. A Game in Strategic Form. A situation in which a normal form game is played at every date is particularly easy to embed. Let $N$ be a set of players, and let $A_{i}$ be the (finite) action set of player $i$. A state is simply an action profile $a=\left(a_{i}\right)_{i \in N}$. Starting from some action 
profile $a$ a coalition $S$ can induce any action profile of the form $\left(a_{S}^{\prime}, a_{-S}\right)$, where $a_{-S}$ is that part of the profile not chosen by members of $S$, and $a_{S}^{\prime}$ is any vector of actions on the part of $S .^{7}$

\subsection{Equilibrium}

A process of coalition formation $(\mathrm{PCF})$ is a transition probability $p$ : $X \times X \rightarrow[0,1]$ (so that $\sum_{y \in X} p(x, y)=1$ for each $\left.x \in X\right)$.

We interpret $p$ as capturing the (possibly stochastic) transitions from one state to another. These transitions will be induced by coalitions who stand to benefit from them (see below). ${ }^{8}$

A PCF $p$ induces a value function $v_{i}$ for each player $i$. This value function captures the infinite horizon payoff to a player starting from any state $x$, under the Markov process $p$. Standard observations tell us that the value function for $i$ must be the unique solution to the functional equation

$$
v_{i}(x, p)=u_{i}(x)+\delta_{i} \sum_{y \in X} p(x, y) v_{i}(y, p) .
$$

We are now in a position to define profitable moves. These will be used to impose restrictions on the process of coalition formation. Fix a PCF $p$, a state $x$, and a coalition $S$. Say that $S$ has a (weakly) profitable move from $x$ (under $p$ ) if there is $y \in F_{S}(x)$ (with $y \neq x$ ) such that $v_{i}(y, p) \geq v_{i}(x, p)$ for all $i \in S$. $S$ has a strictly profitable move from $x$ if there is $y \in F_{S}(x)$ such that $v_{i}(y, p)>v_{i}(x, p)$ for all $i \in S$. Finally, say that a move $y$ is efficient for $S$ if there is no other move for $S$, say $z$, such that $v_{i}(z, p)>v_{i}(y, p)$ for all $i \in S$.

A PCF is an equilibrium process of coalition formation (EPCF) if (i) whenever $p(x, y)>0$ for some $y \neq x$, then there is $S$ such that $y$ is a (weakly) profitable and efficient move for $S$ from $x$, and (ii) if there is a strictly profitable move from $x$, then $p(x, x)=0$ and there is a strictly profitable and efficient move $y$ with $p(x, y)>0$.

Thus a going state is allowed to move to another state only if there is a coalition whose members all agree to move to the new state and cannot find any strictly better alternative state (under the going value functions). Moreover, if there is a strictly profitable move, then the state must change,

\footnotetext{
${ }^{7}$ To be sure, dynamic situations of coalition formation derived from strategic form games are not devoid of conceptual issues. Why is $a_{-S}$ fixed when $S$ moves? One interpretation is that an action profile constitutes a temporarily binding agreement, and at every date some coalition receives the opportunity to costlessly renege on such an agreement.

${ }^{8}$ Notice that the stationarity implicit in the definition of a PCF rules out moves that are history-dependent. Thus we are implictly restricting our attention to schemes that have the Markov property.
} 
and there must be at least one move to a state which is interpretable as a strictly profitable and efficient move for some coalition.

Notice that this definition allows for (but does not insist on) possible changes in state in which the initiating coalition is indifferent to the change. At the same time, the definition does not insist that every strictly profitable move (under the equilibrium $\mathrm{PCF}$ ) be given positive probability. This is true in a particularly stark way of "deterministic" PCFs - to be introduced in Section 4 - in which only one coalition is selected to act at each state which admits some profitable move (even though, in principle, there may be several such moves). ${ }^{9}$

Some further remarks on "efficient moves" are to be found in Appendix 1.

\section{EXISTENCE}

Theorem 3.1. An equilibrium process of coalition formation exists.

Remark. The current theorem extends to state spaces that are countable. Whether existence holds in more general cases remains an open question.

Proof. Denote by $\mathcal{P}$ the set of all possible PCF's. We construct a mapping $\phi: \mathcal{P} \Rightarrow \mathcal{P}$, show that a fixed point exists, and observe that a fixed point of $\phi$ must be an EPCF.

We begin by observing that for every $p \in \mathcal{P}$, a unique value function $v_{i}(x, p)$ exists for each player $i$, satisfying (1). Let $\mathbf{v}_{i}(p)$ denote the vector of payoffs $\left\{v_{i}(x, p)\right\}_{x \in X}, \mathbf{u}_{i}$ the vector of current payoffs $\left\{u_{i}(x)\right\}_{x \in X}$, and $P$ the matrix of transition probabilities (under $p$ ). Then (1) may be immediately rewritten as

$$
\left(I-\delta_{i} P\right) \mathbf{v}_{i}(p)=\mathbf{u}_{i}
$$

Since $\delta_{i} \in(0,1), I-\delta_{i} P$ has a dominant diagonal. This guarantees the unique solvability and continuity of $\mathbf{v}_{i}(p)$ in $p$.

To construct $\phi$, first consider $(x, p)$ such that strictly profitable moves exist; let $Y(x, p)$ be the set of all strictly profitable and efficient moves. For each $y \in Y(x, p)$ there is a coalition $S$ such that $y$ is strictly profitable and efficient for $S$ from $x$ (under $p$ ). Call such a coalition allowable (given $(y, x, p)$ ), and for each allowable coalition $S$ define $\sigma_{S}(y, x, p) \equiv$ $\min _{i \in S}\left[v_{i}(y, p)-v_{i}(x, p)\right]$. Having done so, let $\sigma(y, x, p) \equiv \max _{S} \sigma_{S}(y, x, p)$, where the maximum is taken over allowable coalitions $S$. Now define a

\footnotetext{
${ }^{9} \mathrm{We}$ allude to this restriction again in footnote 14, in the context of Example 4.
} 
probability measure over $Y(x, p)$ - call it $q(x, p)$ — by

$$
q(x, p)[y] \equiv \frac{\sigma(y, x, p)}{\sum_{y^{\prime} \in Y(x, p)} \sigma\left(y^{\prime}, x, p\right)},
$$

Define a correspondence $\Delta(x, p)$ as follows: when strictly profitable moves exist, $\Delta(x, p)=\{q(x, p)\}$. Otherwise, $\Delta(x, p)$ be the collection of all probability measures with support contained in the union of $\{x\}$ and the collection of weakly profitable and efficient moves from $x$ (under $p$ ).

Obviously, $\Delta(x, p)$ is nonempty and convex-valued for each $(x, p)$. Now we claim that it is uhc in $p$ for given $x$. To this end, let $p^{k}$ be some sequence in $\mathcal{P}$ converging to $p$. Study a corresponding sequence $q^{k} \in \Delta\left(x, p^{k}\right)$ and extract a convergent subsequence converging to some $q$ (retain original sequence notation). We claim that $q \in \Delta(x, p)$.

This claim is obviously true if no strictly profitable move exists at $(x, p) \cdot{ }^{10}$ So suppose that a strictly profitable move does exist at $(x, p)$. We note that for any $y \in Y(x, p), \sigma\left(y, x, p^{k}\right) \rightarrow \sigma(y, x, p)$ as $k \rightarrow \infty$. [This is very easy to verify, using the fact that $v_{i}(x, p)$ is continuous in $p$ for every $i$ and $x$.]

In particular, this means that for $k$ large enough, $\Delta\left(x, p^{k}\right)$ is a singleton containing the probability measure $q\left(x, p^{k}\right)$ defined by (2). It also means that $q\left(x, p^{k}\right) \rightarrow q(x, p)$.

We have therefore shown that $\Delta(x, p)$ is nonempty, convex-valued and uhc in $p$ for each $x$. Define $\phi: \mathcal{P} \Rightarrow \mathcal{P}$ by $\phi(p)=\prod_{x \in X} \Delta(x, p)$ for every $p \in \mathcal{P}$. Then, by the arguments above, all the conditions for the Kakutani fixed point theorem are satisfied, and there exists $p^{*} \in \mathcal{P}$ such that $p^{*} \in \phi\left(p^{*}\right)$. It is easy to see that $p^{*}$ satisfies all the conditions of an EPCF.

\section{DETERMINISTIC EQUILIBRIUM PROCESSES}

In this section, we narrow our definition considerably. We then compare this narrow definition with existing concepts, as a way of situating our proposed solution in the perspective of existing literature. To this end, introduce the following definitions. A PCF is deterministic if $p(x, y) \in$ $\{0,1\}$ for all states $x$ and $y$. A state $x$ is absorbing if $p(x, x)=1$. An absorbing $P C F$ is one in which for each state $y$, there is some absorbing state $x$ with $p^{(k)}(y, x)>0$ (for some $k \geq 1$ ), where the notation $p^{(k)}$ describes the $k$-step transition probability derived from $p$ in the usual way. Finally, a PCF has unique limit if it is absorbing and possesses a single absorbing state.

\footnotetext{
${ }^{10} \mathrm{All}$ we need to observe is that if $y$ is strictly profitable for the sequence $\left(x, p^{k}\right)$, then it must be weakly profitable for $(x, p)$.
} 


\subsection{Deterministic PCF's with Unique Limit, and the Core}

A classical solution concept is the core. At first sight, the core appears to be an extremely myopic notion, requiring the stability of a proposed allocation to deviations or blocks by coalitions, but not examining the stability of the deviations themselves. However, it is well known that an extended definition that tests the deviations of coalitions by requiring immunity to further deviations by subcoalitions gives back the core once again [26]. This means that the core automatically embodies a certain degree of farsightedness (insofar as chains of deviations by nested coalitions are concerned). We now show that each element of the core in an arbitrary characteristic function game can be "supported" (in a sense to be made precise below) as the outcome of a deterministic EPCF with unique limit. In other words, the core passes a further consistency test where nested deviations are dispensed with.

We also establish a converse that yields an almost-complete characterization of deterministic EPCF's with unique limit.

Fix a finite set $N$ of players. A (finite) characteristic function is a map $V$ that associates with each coalition $S$ a nonempty finite set of payoff vectors in $\mathbb{R}^{S}$. Normalize so that all payoffs are nonnegative.

A state of a characteristic function is a pair $x=(a, \pi)$, where $\pi$ is some partition of the player set into coalitions, and $a$ is a payoff vector such that $a_{S} \in V(S)$ for any coalition $S \in \pi$.

A strong core state is a state $x=(a, \pi)$ such that there is no coalition $S$ and payoff $b \in V(S)$ with $b \geq a_{S}$ and $b \neq a_{S}$. A weak core state is a state $x=(a, \pi)$ such that there is no coalition $S$ and payoff $b \in V(S)$ with $b \gg a_{S}$. Obviously, a strong core state is a weak core state.

We now embed a characteristic function into an intertemporal model of coalition formation. Let $X$ be the collection of all states of the characteristic function. For each partition $\pi$ of $N$ and each coalition $S$, let $W$ denote the set of left-behind players $\{i \in T \backslash S: T \in \pi, T \cap S \neq \emptyset\}$. Now let $\pi_{S}=\{S\} \cup\left\{T^{\prime} \in \pi: T^{\prime} \cap S=\emptyset\right\} \cup \pi(W)$, where $\pi(W)$ is some arbitrary partition of $W$. [Clearly, $\pi_{S}=\pi$ if (and only if) $S \in \pi$.] Now define $F_{S}(x)$ as any collection of states $y=\left(a^{\prime}, \pi^{\prime}\right)$ such that (a) $\pi^{\prime}=\pi_{S}$, (b) $a_{S}^{\prime} \in V(S)$, and indeed, $\cup_{\left(a^{\prime}, \pi^{\prime}\right) \in F_{S}(x)} a_{S}^{\prime}=V(S)$, (c) $a_{T}^{\prime}=a_{T}$ for all coalitions $T \in \pi$ such that $T \cap S=\emptyset$, and (d) if $a^{1}, a^{2}$ satisfy $\left(a^{i}, \pi^{\prime}\right) \in F_{S}(x)$ for $i=1,2$, then $a_{-S}^{1}=a_{-S}^{2}$.

This unwieldy formalism is easily interpreted in words: a move is available to $S$ if the payoff vector (restricted to $S$ ) is feasible for $S$, if the remaining coalition structure consists of the coalitions that $S$ left untouched and some arbitrary partition of players that $S$ left behind, ${ }^{11}$ if the result-

\footnotetext{
${ }^{11}[19]$ considers two formulations of coalition formation games. A $\Delta$-game considers a situation that players who are left behind by a coalitional deviation $S$ are dissolved
} 
ing payoff vector to all non-deviant players is independent of the particular payoff vector chosen by the deviating coalition, and moreover, if the resulting payoffs to coalitions in $\pi$ which have an empty intersection with $S$ remain the same as before. ${ }^{12}$

We may now state the following proposition.

THEOREM 4.1. Let $x^{*}$ be a strong core state of a characteristic function. Then there is $\delta^{*} \in(0,1)$ such that for any collection of discount factors all in $\left(\delta^{*}, 1\right)$ and any associated intertemporal model of coalition formation, there exists a deterministic EPCF defined on that model with $x^{*}$ as its unique limit.

Proof. We will construct a PCF $p$ as a deterministic mapping from state to state, ultimately leading to $x^{*}$. Let $x^{*}=\left(a^{*}, \pi^{*}\right)$, where $\pi^{*}$ is the coalition structure $\left\{S_{1}^{*}, \ldots S_{K}^{*}\right\}$. Let $x$ be any state.

Case 1. $x=x^{*}$. Set $p(x, x)=1$.

CAse 2. Case 1 does not hold, and there exists a player $i$ such that $\{i\} \notin \pi$, and $a_{i}^{*}>a_{i}$. Pick the smallest index $i$ with this property, and set $p(x, y)=1$, where $y=\left(b, \pi^{\prime}\right) \in F_{i}(x)$ with $b_{i}=\max V(\{i\})$.

CAse 3. Cases 1 and 2 do not hold, and there exists a coalition $S \in \pi^{*}$ such that $a_{i}^{*}>a_{i}$ for all $i \in S$. Pick the smallest index $k$ such that $S_{k}^{*}$ has this property, and set $p(x, y)=1$, where $y=\left(b, \pi^{\prime}\right) \in F_{S_{k}^{*}}(x)$ with $b_{S_{k}^{*}}=a_{S_{k}^{*}}^{*} \cdot$

CAse 4. Cases 1, 2 and 3 do not hold, and there exists a coalition $S \in \pi^{*}$ such that $a_{i}^{*} \geq a_{i}$ for all $i \in S$, and either $S \notin \pi$, or $S \in \pi$ and $a_{S}^{*} \neq a_{S}$. Pick the smallest index $k$ such that $S_{k}^{*}$ has this property, and set $p(x, y)=1$, where $y=\left(b, \pi^{\prime}\right) \in F_{S_{k}^{*}}(x)$ with $b_{S_{k}^{*}}=a_{S_{k}^{*}}^{*}$.

For this construction to be sensible, at least one of the situations described must obtain. To see this, assume that Cases 1-3 do not hold. We show that Case 4 must hold. To this end, pick any coalition $T$ in $\pi$. If it is a singleton, we must have $a_{i} \leq a_{i}^{*}$ (because $a^{*}$ is a core allocation). We claim the same is true of all $i \in T$ even if $T$ is not a singleton.

For if this is false, then $a_{j}>a_{j}^{*}$ for some $j \in T$. But then, because $a^{*}$ is a strong core allocation, there exists $i \in T$ such that $a_{i}<a_{i}^{*}$. Clearly

and each player becomes a singleton. On the other hand, a $\Gamma$-game considers a situation that each of the complementary pieces that $S$ left behind stays together. Since we allow any regrouping of players who are left behind by $S$, our setting allows for both $\Delta$ - and $\Gamma$-games.

${ }^{12}$ Note that, in principle, several correspondences of the form $F_{S}(x)$ may be written down that satisfy this "independence property": our results hold for each one of them. 
$\{i\} \notin \pi$ (because $T \in \pi$ and $T$ is not a singleton). But this means that Case 2 holds, a contradiction.

So we have shown that $a^{*} \geq a$. In particular, for any $S \in \pi^{*}$, we have $a_{S}^{*} \geq a_{S}$. To complete the argument, suppose that for all $S \in \pi$, we have $a_{S}^{*}=a_{S}$. Then $\pi$ cannot equal $\pi^{*}$ (otherwise we would be in Case 1). This means that there must exist $S \in \pi^{*}$ (with $a_{S}^{*} \geq a_{S}$, as already shown) such that $S \notin \pi$. So Case 4 holds whenever Cases 1-3 do not.

Therefore the (deterministic) transition from $x$ to $y$ is well-defined in all cases. It is also easy to see that apart from $x^{*}, x \neq y$ for every other state, and that there are no cycles. It follows that $x^{*}$ is the unique absorbing limit of this PCF.

To complete the proof, we must show that all the conditions of an EPCF are satisfied by this PCF. To assure this, we first choose the threshold value of $\delta^{*}$. For any individual $i$, let $m_{i}$ be the maximal payoff that he enjoys over all states in which he receives less than his core payoff $a_{i}^{*}$. Define $\delta_{i}^{*}$ by $\delta_{i}^{* C} a_{i}^{*}=m_{i}$, where $C$ is the total number of states, and $\delta^{*} \equiv \max _{i \in N} \delta_{i}^{*}$. We take it that the discount factor of every player strictly exceeds this threshold.

Begin with the state $x^{*}$, and consider any move by any coalition $S$ to $x=(a, \pi)$. Let $L$ be the members of $S$ who are no better off in the "static sense" by doing so: $L=\left\{j \in S \mid a_{j} \leq a_{j}^{*}\right\}$. Observe that $L$ is nonempty. Now apply our constructed PCF thereafter. Notice that the payoff to any member of $S$ can only change if some member of $L$ initiates a future move (and indeed, this must happen under the PCF). Let $i \in L$ be one of the first movers from $S$ after the initial move by $S$. Given the PCF, $i$ cannot enjoy any more than his core payoff $a_{i}^{*}$ after this move is made. The same is also true for the intervening period between the first move by $S$ and the later move by $i$. We may conclude that $i$ cannot be strictly better off (relative to the core payoff) by participating in the move by $S$. It follows that at $x^{*}$, no strictly profitable move exists, so we are justified in placing $p\left(x^{*}, x^{*}\right)=1$.

Now consider some state $x \neq x^{*}$. Suppose that we are in case 2. By our condition (d) in the definition of the move correspondence $F_{S}$, and given the definition of our PCF, it only needs to be shown that the stipulated move is profitable. Notice that

$$
v_{i}(x, p)=a_{i}+\delta_{i} v_{i}(y, p)
$$

while

$$
v_{i}(y, p) \geq \frac{\delta_{i}^{C}}{1-\delta_{i}} a_{i}^{*},
$$

by the normalization that all payoffs are nonnegative and the fact that the strong core allocation is reached under the PCF in at most $C$ periods. 
Combining these last two expressions, it is easy to see that

$$
v_{i}(y, p)-v_{i}(x, p) \geq \delta_{i}^{C} a_{i}^{*}-a_{i}>\delta^{* C} a_{i}^{*}-a_{i} \geq m_{i}-a_{i} \geq 0,
$$

where the very last inequality follows from the fact that $a_{i}^{*}>a_{i}$, and the definition of $m_{i}$.

Now suppose that we are in cases 3 or 4 . Then there is some coalition $S_{k}^{*}$ which is required to move directly to its segment of the strong core allocation, creating the state $y$. Moreover, by condition (c) in the definition of the move correspondence $F_{S}$, and given our PCF, $S_{k}^{*}$ will receive this payoff for ever after. Clearly this move is weakly profitable. To see that it is efficient, consider any other state $z=\left(b, \pi^{\prime}\right) \in F_{S_{k}^{*}}(x)$. Following the same line of reasoning as in case 1 , let $L$ be the subset of people in $S_{k}^{*}$ who are no better off (relative to their core payoff) by doing so: $L=\left\{j \in S \mid a_{j} \leq a_{j}^{*}\right\}$. Observe that $L$ is nonempty. Now follow a parallel argument to see that there exists $i \in S_{k}^{*}$ who cannot derive a higher payoff from the route precipitated by this alternative move by $S_{k}^{*}$. In other words, the prescribed move for $S_{k}^{*}$ is efficient.

Finally, notice that our ordering of the cases guarantees that some strictly profitable (and efficient) move is always made whenever one exists.

The following proposition describes an almost-complete converse to Theorem 4.1.

TheOREM 4.2. Fix some characteristic function. There is $\delta^{*} \in(0,1)$ such that for any collection of discount factors all in $\left(\delta^{*}, 1\right)$, and for any deterministic EPCF defined on any associated intertemporal model of coalition formation with $x^{*}$ as its unique limit, $x^{*}$ must be a weak core state.

Proof. Our first task is to fix $\delta^{*}$. For any $x=(a, \pi)$ that is not a weak core state, there is some coalition $S$ and $b \in V(S)$ such that $b \gg a_{S}$. Pick $\epsilon>0$ such that $b \geq \epsilon+a_{S}$. Because there are only a finite number of states, we may choose $\epsilon$ so that this inequality holds uniformly across all noncore states, all coalitions $S$, and all allocations of the form $b$ that do better for $S$. Next, denote by $M$ the maximal (one-period) payoff accruing to any player under the characteristic function. Finally, define $\delta^{*}$ so that $\left(1-\delta^{* C}\right) M<\epsilon$, where $C$ is the total number of states.

Consider any associated intertemporal model of coalition formation, with $\delta_{i}>\delta^{*}$ for all $i \in N$. Suppose, contrary to the statement of the theorem, that there exists a deterministic $\operatorname{EPCF} p$ with unique limit $x$, where $x$ is not a weak core state. Then there is some coalition $S$ and $b \in V(S)$ such that $b \gg a_{S}$. Let $S$ induce the state $y=\left(a^{\prime}, \pi^{\prime}\right) \in F_{S}(x)$ such that $a_{S}^{\prime}=b$. Given the EPCF, starting from $y$, the system must attain $x$ again 
in at most $C$ periods and stay there. Moreover, for this to happen, some member $i$ of $S$ must participate in some profitable move $z$ from $y$ (for if not, all members of $S$ must receive $b$ for ever after, a contradiction to the fact that $x$ is the unique limit). This means that

$$
v_{i}(z, p) \geq v_{i}(y, p)
$$

Now observe that

$$
v_{i}(y, p)=b_{i}+\delta_{i} v_{i}(z, p)
$$

so that

$$
\begin{aligned}
v_{i}(z, p)-v_{i}(y, p) & =\left(1-\delta_{i}\right) v_{i}(z, p)-b_{i} \\
& \leq\left(1-\delta_{i}^{C}\right) M+\delta_{i}^{C} a_{i}-b_{i} \\
& <\epsilon+a_{i}-b_{i} \\
& \leq 0 .
\end{aligned}
$$

But this inequality contradicts (3).

Theorems 4.1 and 4.2, taken together, show that in the context of characteristic functions, the concept of the core and that of a deterministic EPCF with unique limit are (essentially) equivalent, as long as discount factors are taken close enough to unity. Of course, the core does not exist for all games while a deterministic EPCF may exist (see, for instance, Example 7), and a general EPCF certainly does. Moreover, as already noted, there are models of coalition formation which do not come from characteristic functions. In both these cases the concept of an EPCF may provide new insights, as we argue in the later sections of this paper.

At the same time, our core characterization isn't exactly old wine in a new bottle. To appreciate this, notice that Theorems 4.1 and 4.2 would also have gone through if we were to take $\delta$ very close to zero rather than unity. The reason is simple: when $\delta=0$ we have the purely myopic case in which the short-sighted blocking intuitions of the core apply straightforwardly. But - as stated more than once - the novelty of the present case is the results hold when discounting vanishes. It is therefore not surprising to find that a deterministic EPCF (with $\delta$ close to 1 ) may rule out non-core allocations in ways that are strikingly different from those suggested by the standard definition of the core does. To appreciate this, consider the following example. ${ }^{13}$

\footnotetext{
${ }^{13}$ Recall that we have used a general way of transforming characteristic functions to $F_{S}$-correspondences. In all the examples, we use the particular specification that when a new coalition forms, the induced coalition structure (that immediately results) corresponds to the $\Gamma$ formulation in [19]; see our footnote 11 for a definition.
} 
EXAMPLE 4. The following characteristic function is related to the coalition formation game studied in [3] and [8]. $N=\{1,2,3\}, V(1,2,3)=\{(2,2,2)\}$, $V(1,2)=\{(3,3)\}, V(2,3)=\{(4,1)\}$, and $V(S)$ contains only the zero payoff vector for all other coalitions $S$. It is easy to see that this game has a unique core state (coalition structure) $\{\{1\},\{2,3\}\}$.

We now describe a deterministic EPCF with unique limit. Because each coalition structure has only one payoff vector, we may equate states with (the five) coalition structures and schematically write down the PCF as follows:

$x_{1}=\{\{1,2,3\}\} \rightarrow\{2\} x_{4}$
$x_{2}=\{\{1,2\},\{3\}\} \rightarrow_{\{2,3\}} x_{3}$
$\mathbf{x}_{3}=\{\{\mathbf{1}\},\{\mathbf{2}, \mathbf{3}\}\} \rightarrow \mathbf{x}_{3}$
$x_{4}=\{\{1,3\},\{2\}\} \rightarrow_{\{2,3\}} x_{3}$
$x_{5}=\{\{1\},\{2\},\{3\}\} \rightarrow_{\{2,3\}} x_{3}$

It is easy to check that if player 2's discount factor exceeds $1 / 2$, this scheme is indeed an EPCF. Now focus on $x_{1}$. This coalition structure is not a core state. The only blocking coalition is formed by players 1 and 2 . However, if player 1 is farsighted enough, she would not join such a move since she expects that player 2 would "betray" her by forming a move with player 3 to achieve $x_{3}$. That is, she would be better off by not deviating from $x_{1}$ from the first place. The point is that our EPCF does eliminate the non-core state $x_{1}$, but cannot do so by the argument that underlies the definition of the core.

The reason that $x_{1}$ is not stable in the PCF is that player 2 deviates alone, expecting to create a further subsequent move with player 3 . Actually, player 2 suffers from a low payoff for one period right after the unilateral deviation, and enjoys higher payoffs for ever from the next period. Thus, player 2's motive for deviating from $x_{1}$ is really based on her farsightedness. Thus the reason why $x_{1}$ is unstable comes from farsightedness, while under the standard definition, $x_{1}$ is eliminated for an immediate (myopic) gain. These are very different arguments, yet they arrive at the same conclusion. ${ }^{14}$

\footnotetext{
${ }^{14}$ Notice that under our deterministic EPCF, there may be several profitable moves at a particular state. For instance, it is true that at state $x_{1}$, both players 1 and 2 may jointly wish to move if they are given the chance to do so. The reason why 1 also wants to move, in contrast with the argument in the main text, is that if he does not, he foresees disaster coming in the shape of 2 moving anyway, as prescribed by the EPCF. But the point is that the coalition $\{12\}$ is not given the opportunity to move. If we do insist on going all the way with this argument while restraining ourselves to deterministic PCFs, we must allow only $\{12\}$ to move - not just today, but tomorrow as well - but as the text argues, this cannot give rise to an EPCF. [To be sure, there may be stochastic
} 
We end this section by addressing an obvious gap in our characterization. Theorem 4.1 cannot be strengthened to include all weak core states, as the following example shows.

EXAmple 5. Consider the following characteristic function: $N=\{1,2,3\}$, $V(1,2,3)=\{(5,5,5)\}, V(1,2)=\{(5,6)\}, V(1)=V(2)=V(3)=\{1\}$, and $V(S)$ contains only the zero payoff vector for all other coalitions $S$. The coalition structure $\{1,2,3\}$ and its associated payoff vector $(5,5,5)$ represents a weak core state (it is not a strong core state because of the coalition $\{1,2\})$. But this cannot be supported as the unique limit of a deterministic EPCF (for any discount factor less than one). For to move from the structure $\{1,2\},\{3\}$ to the weak core, player 1 must participate in the first move (player 2 does not want to deviate, and player 3 can only do so with the help of players 1 and 2). But it is easy to see that if player 1 participates in any move, he must temporarily receive strictly less than his weak core payoff of 5 and later, no more than 5 . Therefore it does not pay him to deviate for any discount factor less than one.

On the other hand, Theorem 4.2 cannot be strengthened to exclude all states that are not in the strong core, as the following variation on Example 5 shows.

ExAmple 6. $N=\{1,2,3\}, V(1,2,3)=\{(5,5,5)\}, V(1,2)=\{(5,6)\}$, $V(1)=\{5\}$, and $V(S)$ contains only the zero payoff vector for all other coalitions $S$. It is easy to see that the coalition structure $\{1,2,3\}$ and its associated payoff vector $(5,5,5)$ represents a weak core state (it is not a strong core state because of the coalition $\{1,2\}$ ). Consider the deterministic $\mathrm{PCF}$ in which the grand coalition structure is the unique limit, and all states map directly to this structure, except for the structure $\{1,2\},\{3\}$, which is mapped to the structure of singletons. It is easy to check that this is an EPCF.

\subsection{Deterministic Absorbing PCF's and Consistency}

We have seen that the narrowest restrictions imposed so far - deterministic PCF's with unique limit - provide an almost-complete characterization of the core. Now let us loosen the restrictions slightly by dropping the requirement of a unique limit, but nevertheless not permitting any cycles. This gives us the broader class of absorbing deterministic processes of coalition formation (recall the formal definition stated earlier).

We first show that this relaxation permits absorbing states that are disjoint from core states, irrespective of whether or not the core itself is empty.

EPCFs where both coalitions $\{12\}$ and $\{2\}$ obtain the chance to move.] This example therefore also illustrates the conceptual restrictions mentioned at the end of Section 2.3. 
ExAmPle 7. Consider the following two characteristic function games. Game 1 is as follows: $N=\{1,2,3,4\}, V(1,2,3,4)=\{(4,3,2,2)\}, V(2,3,4)=$ $\{(4,3,5)\}, V(1,3)=\{(2,4)\}, V(1,4)=\{(3,4)\}, V(2,4)=\{(2,3)\}, V(i)=$ 1 for any other $i \in N$, and $V(S)$ contains only zero payoffs for all other coalition $S$. Game 2 retains all the features of Game 1 , but changes $V(2,3,4)$ to $\{(4,3,4)\}$ and $V(1,4)$ to $\{(3,5)\}$. Game 1 does not have any core state (weak or strong), and Game 2 has a unique (weak and strong) core allocation $\{\{1,4\},\{2\},\{3\}\}$.

There are fifteen states in each of these two games, since each coalition structure has only one payoff vector. Define an absorbing deterministic $\mathrm{PCF}$ in the following schematic way:

$$
\begin{aligned}
& \mathbf{x}_{1}=\{\{\mathbf{1}, \mathbf{2}, \mathbf{3}, \mathbf{4}\}\} \rightarrow \mathbf{x}_{1} \\
& x_{2}=\{\{1,2,3\},\{4\}\} \rightarrow_{\{1,2,3,4\}} x_{1} \\
& x_{3}=\{\{1,2,4\},\{3\}\} \rightarrow_{\{1,2,3,4\}} x_{1} \\
& x_{4}=\{\{1,3,4\},\{2\}\} \rightarrow_{\{2,4\}} x_{8} \\
& x_{5}=\{\{2,3,4\},\{1\}\} \rightarrow_{\{1,3\}} x_{8} \\
& x_{6}=\{\{1,2\},\{3,4\}\} \rightarrow_{\{1,2,3,4\}} x_{1} \\
& x_{7}=\{\{1,2\},\{3\},\{4\}\} \rightarrow_{\{1,3\}} x_{9} \rightarrow_{\{2,4\}} x_{8} \\
& \mathbf{x}_{8}=\{\{\mathbf{1}, \mathbf{3}\},\{\mathbf{2}, \mathbf{4}\}\} \rightarrow \mathbf{x}_{8} \\
& x_{9}=\{\{1,3\},\{2\},\{4\}\} \rightarrow_{\{2,4\}} x_{8} \\
& x_{10}=\{\{1,4\},\{2,3\}\} \rightarrow_{\{1\}} x_{12} \rightarrow_{\{1,2,3,4\}} x_{1} \\
& x_{11}=\{\{1,4\},\{2\},\{3\}\} \rightarrow_{\{1\}} x_{15} \rightarrow_{\{1,2,3,4\}} x_{1} \\
& x_{12}=\{\{2,3\},\{1\},\{4\}\} \rightarrow_{\{1,2,3,4\}} x_{1} \\
& x_{13}=\{\{2,4\},\{1\},\{3\}\} \rightarrow_{\{1,3\}} x_{8} \\
& x_{14}=\{\{3,4\},\{1\},\{2\}\} \rightarrow_{\{1,2,3,4\}} x_{1} \\
& x_{15}=\{\{1\},\{2\},\{3\},\{4\}\} \rightarrow_{\{1,2,3,4\}} x_{1}
\end{aligned}
$$

The absorbing states under this PCF are $x_{1}$ and $x_{8}$. We claim that if the common discount factor $\delta$ satisfies $\delta \geq \frac{3}{4}$, then this is actually an EPCF. States $x_{10}$ and $x_{11}$ (involving a coalition $\{1,4\}$ ) are not an absorbing state, since player 1 wants to break off to generate state $x_{1}$ if $\delta \geq \frac{3}{4}$. Now, between the two absorbing states, players 1 and 2 prefer $x_{1}$ to $x_{8}$, and players 3 and 4 prefer $x_{8}$ to $x_{1}$. However, starting from $x_{1}$, players 3 and/or 4 can move only to $x_{2}, x_{3}$, and $x_{6}$. All of these states will come back to $x_{1}$. Thus, players 3 and 4 cannot move the state to $x_{8}$ without the help of players 1 and/or 2. A parallel argument applies to players 1 and 2 at $x_{8}$, if they try to go to $x_{1}$. There is no temporal gain from those moves, either (given $\delta \geq \frac{2}{3}$ ). We have therefore shown that there may be an absorbing deterministic EPCF with no core elements among its absorbing states, and this is true regardless of whether the core is empty or not.

Is this a counterexample to our earlier theorems on core equivalence? No, it is not, for this EPCF has multiple absorbing states. Example 7 tells 
us it is possible to "support" a non-core state as an absorbing state, by knocking out possible blocks or deviations by further moves to some other absorbing state.

Combining this observation with the results of the previous section, we see that the core does possess a nice consistency property in a "selfreferential" sense: a deterministic EPCF with a unique limit picks out a core point. But when the self-referential nature of the process is dropped (by admitting more than one absorbing state), then the possibilities widen beyond the core.

It turns out, however, that all absorbing deterministic EPCF's have absorbing states that lie within the largest consistent set. This notion is due to Chwe [11]. Consider any model of intertemporal coalition formation (not necessarily one derived from a characteristic function) Following Chwe, say that a state $y$ indirectly dominates some other state $x$, if there exist $x^{0}, x^{1}, \ldots, x_{m}$ in $X$ with $x^{0}=x$ and $x^{m}=y$, and coalitions $S_{0}, S_{1}, \ldots, S_{m-1}$ such that for $j=0, \ldots, m-1, x^{j+1} \in F_{S_{j}}\left(x^{j}\right)$ and $u_{i}(y) \geq u_{i}\left(x^{j}\right)$ for all $i \in S_{j}{ }^{15}$

Notice how the concept of indirect domination makes reference to payoff comparisons between each intermediate state in the "domination chain" and the final outcome $y$. It is in this sense that the concept of indirect domination incorporates farsightedness.

Now say that a collection $Y$ of states is consistent if the following holds: $x \in Y$ if and only if for every coalition $S$ and for any state $z \in F_{S}(x)$, there exists $y \in Y$, where either $y=z$ or $y$ indirectly dominates $z$, such that the inequality $u_{i}(x) \geq u_{i}(y)$ holds for at least one player $i \in S$.

In other words, a collection of states is consistent if every coalitional move from any element of that collection leads to a "domination chain" (starting with the move and ending within the given collection of states) such that at the "end" of that chain, there is some member of the original deviating coalition who feels that the move was not worthwhile.

Proposition 1 in [11] establishes that there is a largest consistent set among all consistent sets: a set which is itself consistent and which contains every consistent set. ${ }^{16}$ The following proposition links (at least in

\footnotetext{
${ }^{15}$ Our definition of indirect domination requires that all players be weakly better off. Chwe's definition requires that they all be strictly better off. Given our insistence on efficient moves, this will not turn out to be a major issue. In any case, notice that there is no inconsistency between the use of weak inequalities both as a potential force for movement by coalitions, as well as its use as a deterrent for movement. Indifference may be resolved in either direction.

${ }^{16}$ As noted in footnote 15 , we use the weak domination ordering, but Chwe's proposition extends to this case with no changes.
} 
one direction) the largest consistent set to the limit states of absorbing deterministic EPCF's. ${ }^{17}$

TheOREM 4.3. There exists $\delta^{*} \in(0,1)$ such that for any collection of discount factors all in $\left(\delta^{*}, 1\right)$, and for any absorbing deterministic EPCF, the set of all absorbing states is contained within the largest consistent set.

Proof. Let $C$ be the total number of states. Let $M$ and $W$ be the maximal and minimal (one-period) payoffs to any player. Pick $\delta^{*} \in(0,1)$ such that for any two states $x$ and $y$ in $X$ and any index $i$ with $u_{i}(x)>u_{i}(y)$, we have (i) $u_{i}(x)>\left(1-\delta^{* C}\right) M+\delta^{* C} u_{i}(y)$, and (ii) $\left(1-\delta^{* C}\right) W+\delta^{* C} u_{i}(x)>$ $u_{i}(y)$. Consider any collection of discount factors all in $\left(\delta^{*}, 1\right)$, and fix some absorbing deterministic EPCF. Let $Z \subseteq X$ be its set of absorbing states.

Let $z \in Z$ be some absorbing state. Fix any coalition $S$ and consider any $x \in F_{S}(z)$. Use the notation $x^{0}, x^{1}, \ldots, x^{m}$ to describe the subsequent path prescribed by the PCF starting from $x=x^{0}$ and ending at the absorbing state $x^{m}=y \in Z$. Because the PCF is an equilibrium, we also know that there are coalitions $S_{0}, S_{1}, \ldots, S_{m-1}$ such that for $j=0, \ldots, m-1$, $x^{j+1} \in F_{S_{j}}\left(x^{j}\right)$ and

$$
v_{i}\left(x^{j+1}\right) \geq v_{i}\left(x^{j}\right)
$$

for all $i \in S_{j}$. Now observe that $v_{i}\left(x^{j}\right)=u_{i}\left(x^{j}\right)+\delta_{i} v_{i}\left(x^{j+1}\right)$, so that by (4),

$$
\left(1-\delta_{i}\right) v_{i}\left(x^{j+1}\right) \geq u_{i}\left(x^{j}\right)
$$

for each $S_{j}$ and $i \in S_{j}$. Next, note that

$$
\left(1-\delta_{i}\right) v_{i}\left(x^{j+1}\right) \leq\left(1-\delta_{i}^{C}\right) M+\delta_{i}^{C} u_{i}(y)
$$

(because the PCF from $x^{j+1}$ leads to the absorbing state $y$ in at most $C$ steps), and combining this with (5), we may conclude that

$$
\left(1-\delta_{i}^{C}\right) M+\delta_{i}^{C} u_{i}(y) \geq u_{i}\left(x^{j}\right) .
$$

But this means (by (i) in our definition of $\delta^{*}$ ) that

$$
u_{i}(y) \geq u_{i}\left(x^{j}\right)
$$

\footnotetext{
${ }^{17}$ We thank an anonymous referee for pointing out an error in an earlier version of this theorem.
} 
for all $S_{j}$ and all $i \in S_{j}$. (6) proves that $y$, apart from being in $Z$, is an indirect objection to $x$.

Moreover, since $x$ is a possible move (by $S$ ) from $z$ and $z$ is an absorbing state, $v_{i}(x) \leq v_{i}(z)$ for some $i \in S$. Because $z$ is absorbing, it follows that

$$
\left(1-\delta_{i}\right) v_{i}(x) \leq u_{i}(z)
$$

Now observe that

$$
\left(1-\delta_{i}\right) v_{i}(x) \geq\left(1-\delta_{i}^{C}\right) W+\delta_{i}^{C} u_{i}(y)
$$

(because the PCF from $x$ leads to the absorbing state $y$ in at most $C$ steps), and combining this with (7) we may conclude that

$$
u_{i}(z) \geq\left(1-\delta_{i}^{C}\right) W+\delta_{i}^{C} u_{i}(y)
$$

By part (ii) in the definition of $\delta^{*}$, we deduce that

$$
u_{i}(z) \geq u_{i}(y)
$$

for some $i \in S$.

Now (6) and (8) together prove that $f(Z) \supseteq Z$, where $f(Z)$ is the set of all states $x$ such that for every coalition $S$ and for any state $y \in F_{S}(x)$, there exists $z \in Z$, where either $y=z$ or $z$ indirectly dominates $y$, such that the inequality $u_{i}(x) \geq u_{i}(z)$ holds for some $i \in S$. Using the same argument in the proof of Proposition 1 in [11], we may conclude that $Z$ is contained in the largest consistent set.

It is worth noting that the largest consistent set may be "large" but is certainly not exhaustive. For instance, in the Prisoners' Dilemma transformed into a dynamic model of coalition formation along the lines of Example 3 - the largest consistent set is a singleton consisting of the cooperative outcome. Nevertheless, there are reasons to believe that the largest consistent set may be too inclusive in some situations (see, for example, the discussion in [34]). The notion of an EPCF highlights one reason for this, as elaborated in the following example.

Example 8. $\quad N=(1,2,3)$. There are four states, represented by the payoffs they provide to each of the three players: $x_{1}=(2,2,2), x_{2}=$ $(0,0,0), x_{3}=(6,6,0)$ and $x_{4}=(1,0,6)$. We describe the correspondence $F_{S}$ as follows. At $x_{1}$, the coalition $\{12\}$ or player 3 are the only coalitions that can move, and the only move (in either case) is to $x_{2}$. At $x_{2}$, only coalitions $\{2\}$ and $\{13\}$ can move, and in both case either $x_{3}$ or $x_{4}$ may be induced at will. From no other state is any move possible, and no other coalition is capable of any other move. 
It is easy to see that the largest consistent set consists of the three states $\left(x_{1}, x_{3}, x_{4}\right)$. In particular, the state $x_{1}$ is a member of this set for the following reason: the coalition $\{12\}$ avoids inducing $x_{2}$ because it anticipates the continuation by $\{13\}$ to $x_{4}$, and player 3 similarly negates a move to $x_{2}$ because she fears the subsequent creation of state $x_{3}$ (by player 2).

However, there is no deterministic absorbing EPCF - and indeed, no EPCF at all - with $x_{1}$ as an absorbing state (provided that discount factors are close enough to unity). To see this, let $p$ be the probability that some EPCF assigns to $\{2\}$ moving at $x_{2}$ (so that $1-p$ is asigned to $\{13\}$ ). Neglecting discounting for a moment, note that if $p>1 / 3$, then $\{12\}$ will want to move from $x_{1}$, whereas if $p<2 / 3$ player 3 will want to move from $x_{1}$. It is now trivial to see that the for discount factors close enough to 1 , $x_{1}$ cannot be an absorbing state.

This example shows quite starkly why the notion of consistency is less restrictive than that of an EPCF. Two domination chains along two indirect objections may have different moves attached to them starting from the same state. Thus, as seen above, in the largest consistent set, coalition $\{12\}$ entertains one sort of conjecture about what will happen at $x_{2}$ and player 3 entertains another. If all players have common beliefs (as they must in an EPCF), then this possibility cannot arise. This is one reason why the set of all absorbing states (under all deterministic absorbing EPCFs) is typically a strict subset of the largest consistent set. ${ }^{18}$

\subsection{Deterministic Schemes: Absorption, Cycles and Efficiency}

Example 8 in the previous section makes the point that the set of absorbing states (under deterministic absorbing EPCFs) can be a strict subset of the largest consistent set. It does so by pruning inefficient outcomes from that set. This suggests that our dynamic structure may be generally adept at taking out inefficient outcomes. Certainly, this is true of absorbing schemes that have unique limit (and discount factors close to unity), at least in the space of characteristic functions, by virtue of Theorem 4.2. For schemes with multiple absorbing states, this is not true.

Insert Figure 1 approximately here.

Example 9. $N=\{1,2\}, X=\{a, b, c, d\}$, and $F$ is described in Figure 1. In the class of absorbing deterministic schemes there is exactly one equilibrium, provided the discount factor of each player exceeds $2 / 3$. This equilibrium has absorbing states $\{a, c\}$. Notice that the payoffs from these states are inefficient. ${ }^{19}$

\footnotetext{
${ }^{18}$ Another reason has to do with the efficiency of coalitional moves, something we do not stress here. But see [34], in which similar concerns are raised in the context of a static model of coalitional moves.

${ }^{19}\{a, c\}$ is also the largest consistent set.
} 
To see why, first note that in any absorbing deterministic equilibrium, neither $b$ nor $d$ can be absorbing states. For suppose, on the contrary, that $b$ is absorbing. Then notice that $a$ cannot be absorbing; indeed, that $a \rightarrow b$. This means that a move from $b$ to $c$, engineered by player 2 , has the following possible payoff continuations for player 2 : the constant payoff $(2,2, \ldots)$, the path $(2,6,6, \ldots)$, and the path $(2,6,0,1,1 \ldots)$. In each of these cases 2 earns a (normalized) discounted payoff that exceeds 1 , which contradicts the presumption that $b$ is absorbing. The argument that $d$ cannot be absorbing is an exact parallel.

So $a$ or $c$ (or both) must be absorbing, and there are no other absorbing states.

Next, observe that both $a$ and $c$ must simultaneously be absorbing. For say only $a$ were absorbing. Then it must be the case that $d \rightarrow a$. Now consider a move from $a$ to $b$, engineered by $\{1,2\}$. It is obvious that player 2 must earn positive payoff from this move. Moreover, for any $\delta_{1}<1$, player 1's (normalized) discounted payoff must strictly exceed 2 (we use here the observation that $d \rightarrow a$ ). This contradicts the assumption that only $a$ is absorbing. A parallel argument holds for $c$. Therefore both $a$ and $c$ are absorbing.

This leaves us with only one possible absorbing deterministic EPCF, in which $d \rightarrow a$ and $b \rightarrow c$. Indeed, such a PCF is an equilibrium, provided that the discount factor of each player exceeds $2 / 3$. The outcome is inefficient.

Readers interested in understanding better the source of this efficiency failure are referred to Appendix 1, item 5.

To conclude this section, consider the following PCF which, while deterministic, has no absorbing states: $a \rightarrow b \rightarrow c \rightarrow d \rightarrow a$. Provided that discount factors are close to unity, it is easy to check that each move prescribed by the scheme is strictly profitable (and efficient in the class of all profitable moves). Therefore this cyclical scheme is an EPCF. For discount factors close to one, the (normalized) discounted payoff to each player is approximately 2.25. This payoff vector is efficient. In what follows, we move on to a closer investigation of cyclical and stochastic schemes.

\section{STOCHASTIC EQUILIBRIUM PROCESSES}

In the remainder of the paper, we concentrate on stochastic processes of coalition formation. Uncertainty enters the story in two distinct ways. First, at any stage, several coalitions may have profitable moves. Which coalition gets to move may well be probabilistically chosen. Second, it is possible that a particular coalition has more than one efficient move, and that it might randomize among them. The discussion that follows shows 
that in many typical situations one or more of these randomizations may be necessary in order to generate an equilibrium.

The uncertain nature of the process may or may not be intertwined with cycles - possibly stochastic reversions of the state of the game to some given state. Formally, a (nonsingleton) collection of states $\left(x^{1}, \ldots, x^{k}\right)$ under a PCF forms a (stochastic) cycle if $p\left(x^{i}, x^{i+1}\right)>0$ for all $1 \leq i<k$ and $p\left(x^{k}, x^{1}\right)>0$. A PCF that exhibits a cycle will be called cyclical.

The purpose of the analysis that follows is to understand these phenomena, largely through the use of examples. A large part of our discussion will take place explicitly in the context of strategic form games.

\subsection{Randomization and Cycles: An Example}

The purpose of this section is to illustrate the "need" for cycles and/or randomization in certain situations. We do this by considering the following restatement of the "roommate problem". This is a situation with three players, any of two of whom can share a room. In each case, the player left out obtains zero. Moreover, for each pair of roommates, there is one who obtains a payoff of 1 , while the other obtains a payoff of $a$ (to be parametrically varied in the example). Details follow, couched in the language of a model of coalition formation.

Example 10. Let $N=\{1,2,3\}, X=\{x, y, z\}, F_{\{2,3\}}(x)=\{x, y\}$, $F_{\{1,3\}}(y)=\{y, z\}, F_{\{1,2\}}(z)=\{z, x\}$, and $F_{S}\left(x^{\prime}\right)=\left\{x^{\prime}\right\}$ for all other combinations of $\left(x^{\prime}, S\right)$. Players have a common discount factor $\delta$. Payoffs for each state $x^{\prime}$ are described in the following array:

\begin{tabular}{|c|c|c|c|}
\hline$u_{i}\left(x^{\prime}\right)$ & $x$ & $y$ & $z$ \\
\hline 1 & 1 & 0 & $a$ \\
\hline 2 & $a$ & 1 & 0 \\
\hline 3 & 0 & $a$ & 1 \\
\hline
\end{tabular}

Note that it is easy to rewrite this example in the more familiar characteristic function form. ${ }^{20}$ Appendix 2 contains a demonstration of the following

OBSERVATION 1. The game in Example 10 admits the following unique EPCF that is symmetric for any $a$ and $\delta$ : For $a \leq \frac{1}{1+\delta}, p(x, y)=p(y, z)=$ $p(z, x)=1$, and for $a>\frac{1}{1+\delta}, p(x, y)=p(y, z)=p(z, x)=\frac{(1-a)(1-\delta)}{\delta(2 a-1)}$.

\footnotetext{
${ }^{20}$ Let $N=\{1,2,3\}$ with $V(\{1,2,3\})=\{(0,0,0)\}, V(\{1,2\})=\{(1, a)\}, V(\{2,3\})=$ $\{(1, a)\}, V(\{3,1\})=\{(1, a)\}$, and $V(\{i\})=\{0\}$ for any $i \in N$. Then the relevant part of the game is described as the game in Example 9. We can construct a strategic form representation of essentially the same game.
} 
This example (and the accompanying Observation) is designed to illustrate several points.

First, there is no hope of a general existence result for deterministic schemes. This is true of characteristic functions for which the core is empty (as the example demonstrates). While it is also true of strategic form games, we will also see situations in which stochastic EPCF's coexist with their deterministic counterparts, leading to new insights.

Second, observe that once cycles and randomization make an appearance, the cardinality of payoffs really matters in the determination of a particular equilibrium. In the example, if $a$ is small enough (that is, $a \leq \frac{1}{1+\delta}$ ), then the equilibrium cycle is deterministic, and there is no chance of remaining in the same state in any period. However, as $a$ goes up from $a=\frac{1}{1+\delta}$, the probability of moving to the next state comes down. The cycles turn stochastic. As $a$ increases, each coalition structure becomes relatively more stable since the cyclical movement becomes slower in the stochastic sense, although no state ever becomes an absorbing state. On the other hand, given $a>\frac{1}{1+\delta}, p$ goes down as $\delta$ increases. If $\delta$ is very close to unity, $p$ is very close to zero. Notice that $p$ never becomes zero - the tension of a possible move is needed to sustain the scheme. ${ }^{21}$ Nevertheless, we still can say that if $\delta$ goes to unity, each coalition structure becomes more stable in a stochastic sense.

Third, the example illustrates one of the two sources of stochastic behavior discussed earlier. At each state, there is only one potential deviating coalition. Yet an EPCF can (and sometimes, as in the example, must) be stochastic. Randomization occurs not over multiple deviating coalitions, but over whether a single coalition moves or stays. This type of randomization can occur only when at least one member of the deviating coalition needs to be indifferent between moving and staying. Note that in such a case, the payoff of a player who is indifferent between these options has a very simple form. For instance, suppose that player 1 is indifferent between deviating and staying at state $z$ in the EPCF when $a>\frac{1}{1+\delta}$ $\left(p(z, x)=\frac{(1-a)(1-\delta)}{\delta(2 a-1)}\right)$. Then it must be the case that ${ }^{22}$

$$
v_{1}(z, p)=v_{1}(x, p)=\frac{a}{1-\delta}
$$

This property is not specific to the roommate problem. As long as (i) there is only one possible coalitional deviation, and (ii) that coalitional

\footnotetext{
${ }^{21}$ It should be noted, however, that asymmetric roommate problems (in which the cardinalities of vNM utility functions or the values of discount factors differ across agents) may have well absorbing states.

${ }^{22}$ The claim to be made follows from the fact that $v_{1}(z, p)=a+$ $\delta\left[p(z, x) v_{1}(x, p)+(1-p(z, x)) v_{1}(z, p)\right]=a+\delta v_{1}(z, p)$ (we have $v_{1}(x, p)=v_{1}(z, p)$ by indifference).
} 
move is randomized, the indifferent player's payoff is exactly the same as the discounted sum of atemporal payoffs from the current state.

In the next section, we will see several examples of the second source of uncertainty: that stemming from randomization over multiple coalitional deviations.

\subsection{Games in Strategic Form}

In this section, we apply our solution concept to strategic form games. Such games are usually employed to describe purely noncooperative situations, but there is no reason why this should necessarily be the case. It is possible that subgroups (or even the entire set) of players can come together to write temporarily binding agreements. Under this interpretation, the period length is the duration for which a binding agreement can be written.

We assume that every member in a coalition needs to agree on a temporary binding agreement. This unanimity postulate is natural in defining a coalitional move (recall, for instance, the definitions of strong Nash and coalition-proof Nash equilibria). Models of binding agreements also use the unanimity principle very widely (see, for instance, the survey [7] and the many references contained therein).

The objective of our analysis is to show how the possibly stochastic nature of coalition formation affects efficiency in strategic-form games.

\subsubsection{Games with Common Payoffs}

It will be useful to begin with a situation in which efficiency is not impaired, and this will serve as a benchmark for the more interesting cases to follow. To this end, consider the class of all strategic games with common payoffs, which yield similar payoffs to all players for any action profile. To be sure, such games are not without genuine strategic significance; for instance, the following well-known pure coordination game (with $a$ and $b$ negative) is a special case:

\begin{tabular}{|c|c|c|}
\hline & $L$ & $R$ \\
\hline$T$ & 1,1 & $b, b$ \\
\hline$B$ & $a, a$ & 0,0 \\
\hline
\end{tabular}

Formally, consider a strategic game with finite player set $N$. Player $i$ has finite action set $A_{i}$. Let $A \equiv \prod_{i \in N} A_{i}$. Player $i$ has payoff function $u_{i}$ defined on $A$. We assume that for each action profile $a \in A$, and for all $i$ and $j, u_{i}(a)=u_{j}(a)$. For simplicity of notation, we assume that there is a unique action profile $a^{*}$ at which all players' payoffs are maximized. We call this game a game of common payoffs. 
It is easy enough (as in Example 3) to embed this game into an intertemporal model of coalition formation. A state will simply describe the ongoing action profile, and $F_{S}(x)$ is the set of all states $a^{\prime}$ such $a_{S}^{\prime} \in \prod_{i \in S} A_{i}$, and $a_{i}^{\prime}=a_{i}$ for all $i \notin S$.

In words: an action vector is available to $S$ if it is feasible for its members and if the remaining players leave their actions unchanged. ${ }^{23}$

To complete the description, assume each player $i$ has a common discount factor $\delta \in(0,1)$.

THEOREM 5.1. Every EPCF for the game of common payoffs with a common discount factor $\delta$ involves $p\left(a^{*}, a^{*}\right)=1$ and has $a^{*}$ as the unique absorbing limit starting from any $a \in A$.

Remark. That at least one such EPCF exists with the claimed property is trivially true. The extra bite of this result lies in its assertion for every EPCF.

Proof. First we prove that $p\left(a^{*}, a^{*}\right)=1$. Suppose not. Then there is some coalition $S$ and a move to a state $a$ such that $v_{i}(a, p) \geq v_{i}\left(a^{*}, p\right)$ for all $i \in S$. Choose $a^{\prime}$ to be some state having the lowest value of $v_{i}$ among all states satisfying the requirement of the previous sentence. [By the assumption of common payoffs and a common discount factor, the same state can achieve this for every player.] By not moving, each member $i$ of coalition $S$ gets a payoff of

$$
u_{i}\left(a^{*}\right)+\delta \sum_{a \in A} p\left(a^{*}, a\right) v_{i}(a, p)
$$

which is obviously larger than $v_{i}\left(a^{\prime}, p\right)$, a contradiction.

Next, we show that $a^{*}$ is the unique absorbing limit. To this end, we first note that if $a^{\prime} \neq a^{*}$, then there exists $a^{\prime \prime} \neq a^{\prime}$ such that $p\left(a^{\prime}, a^{\prime \prime}\right)>0$. Suppose not; then $p\left(a^{\prime}, a^{\prime}\right)=1$. In particular, $v_{i}\left(a^{\prime}, p\right)=\frac{1}{1-\delta} u_{i}\left(a^{\prime}\right)$, while $v_{i}\left(a^{*}, p\right)=\frac{1}{1-\delta} u_{i}\left(a^{*}\right)$. However, since $u_{i}\left(a^{*}\right)>u_{i}\left(a^{\prime}\right)$, we have $v_{i}\left(a^{*}, p\right)>$ $v_{i}\left(a^{\prime}, p\right)$ for any $i \in N$. So there is a strictly profitable move from $a^{\prime}$, which contradicts requirement (ii) of an EPCF.

Now, if $a^{*}$ is not the unique absorbing limit, then the set $C(p) \equiv\{a \in$ $A$ : for any $\left.k \geq 1, p^{(k)}\left(a, a^{*}\right)=0\right\}$ is nonempty. By the common payoff assumption, there exists $a^{\prime} \in C(p)$ such that $v_{i}\left(a^{\prime}, p\right) \geq v_{i}(a, p)$ for any

\footnotetext{
${ }^{23}$ Note that we are economizing on the definition of a state here. We could record the coalition structure that is implicitly in place at every situation. It would make no difference to the results that follow, but the statement would be more cumbersome.
} 
$a \in C(p)$. By the argument of the previous paragraph, there is a state $a^{\prime \prime}$ such that $p\left(a^{\prime}, a^{\prime \prime}\right)>0$.

In order to satisfy requirement (i) of an EPCF, it must be (recalling common payoffs) that $v_{i}\left(a^{\prime}, p\right) \leq v_{i}\left(a^{\prime \prime}, p\right)$ for all $i$. But it is obvious that $a^{\prime \prime} \in C(p)$. Consequently, from the definition of $a^{\prime}$ it follows that $v_{i}\left(a^{\prime}, p\right)=$ $v_{i}\left(a^{\prime \prime}, p\right)$, and indeed, this is true for any state $a^{\prime \prime}$ such that $p\left(a^{\prime}, a^{\prime \prime}\right)>0$.

At the same time, we know that $v_{i}\left(a^{*}, p\right)=\frac{1}{1-\delta} u_{i}\left(a^{*}\right)>v_{i}\left(a^{\prime}, p\right)$. Therefore, we conclude that although $v_{i}\left(a^{*}, p\right)>v_{i}\left(a^{\prime}, p\right)$ for any $i \in N$ (common payoffs), $p\left(a^{\prime}, a^{\prime \prime}\right)>0$ occurs only for $a^{\prime \prime}$ with $v_{i}\left(a^{\prime \prime}, p\right)=v_{i}\left(a^{\prime}, p\right)$. This violates requirement (ii) of an $\mathrm{EPCF}$, a contradiction.

Although games with common payoffs are special, this result provides a strong base for our later remarks. In addition, these results may be of intrinsic interest for coordination games. For instance, [22] contains a related result (see also Corollary 2 in [21]). [22] studies repeated pure coordination games in which only one player can change her action in each period, and show that for $\delta$ close to unity there is a unique subgame perfect equilibrium in which the action profile converges to the Pareto efficient one. To be sure, there are important differences, not the least of which is that our approach permits the writing of temporarily binding agreements.

Binding agreements notwithstanding, the finding of ubiquitous cooperation in common-payoff situations does not extend, even to coordination games with non-common payoffs. The following example describes a $2 \times 2$ game in which there is an EPCF with an inefficient absorbing limit. ${ }^{24}$

EXAMPle 11. Consider the following $2 \times 2$ strategic form game:

\begin{tabular}{|c|c|c|}
\hline & $L$ & $R$ \\
\hline$T$ & 1,1 & $-5,-1$ \\
\hline$B$ & $-1,-5$ & 0,0 \\
\hline
\end{tabular}

Denote $(T, L),(B, L),(T, R),(B, R)$ by $x, y, z$, and $w$, respectively. Assume a common discount factor $\delta$ for both players. Then the game induced by this strategic form game has a EPCF with its unique absorbing limit $w$ if $\delta \geq \frac{2}{3}$.

\footnotetext{
${ }^{24}$ Equilibrium selection in [21] is related to the risk-dominance of an action profile [18], and in the example, something similar plays an important role. Indeed, in the example, the Pareto superior Nash equilibrium is a risk dominated equilibrium $(1-(-1)<0-$ $(-5)$ ). However, in general, the conditions for a breakdown in cooperation are different even in coordination games.
} 
To see this, consider the following PCF: $p(x, y)=p(x, z)=\frac{1}{2}, p(y, w)=$ $p(z, w)=p(w, w)=1$. Then, we have:

$$
\begin{aligned}
& v_{1}(x, p)=v_{2}(x, p)=1+\delta\left(\frac{-1-5}{2}\right)=1-3 \delta, \\
& v_{1}(y, p)=v_{2}(z, p)=-1, \\
& v_{1}(z, p)=v_{2}(y, p)=-5, \\
& v_{1}(w, p)=v_{2}(w, z)=0 .
\end{aligned}
$$

As we can easily see from these expressions, there is an incentive for either player to deviate from $x$ as long as $1-3 \delta \leq-1$, which is equivalent to $\delta \geq \frac{2}{3}$. So the PCF is an EPCF under this condition.

The striking feature of this EPCF is that although $x$ is the highest payoff state for every player, it is not stable. The temporary agreement $x$ is upset by unilateral deviations, in which each deviation is bolstered by the fear of the other player's deviation. Notice that this sort of "meta coordination failure" relies intimately on the failure of common payoffs.

Note, moreover, that this EPCF represents an example of the second type of uncertainty and its effects. Randomization among profitably deviating coalitions may cause inefficiency in the resulting outcome. ${ }^{25}$

\subsubsection{The Prisoners' Dilemma}

The prisoners' dilemma represents a leading example of intrinsic interest. We therefore study the EPCFs of this game in some detail. Consider the following $2 \times 2$ strategic form game:

\begin{tabular}{|c|c|c|}
\hline & $L$ & $R$ \\
\hline$T$ & 1,1 & $b, a$ \\
\hline$B$ & $a, b$ & 0,0 \\
\hline
\end{tabular}

where $a>1$ and $b<0$. As in Example 11, denote $(T, L),(B, L),(T, R)$, $(B, R)$ by $x, y, z$, and $w$ respectively.

Unlike coordination games, $x$ no longer attains the highest possible payoff, and it is well-known that $w$ is the unique dominant strategy Nash equilibrium of this game. Our model of coalition formation yields a more varied set of results, which we attempt to characterize in the following

\footnotetext{
${ }^{25}$ Of course, there are other EPCFs: for instance, a "cooperative" EPCF with $p(x, x)=p(y, x)=p(z, x)=p(w, x)=1$ exists for any value of $\delta$. What Example 11 says is that there can be another EPCF that attains a Pareto inferior state as the unique absorbing state even in a coordination game unless we have common payoffs.
} 
ObServation 2. The Prisoners' Dilemma admits various EPCFs depending on specific parameter values:

\section{Deterministic EPCFs}

(i) there is a deterministic EPCF with its unique absorbing limit at $x$ $(p(x, x)=p(y, w)=p(z, w)=p(w, x)=1)$, iff $a \leq 1+\delta$,

(ii) there is a deterministic EPCF with its unique limit at $w(p(x, y)=$ $p(y, w)=p(z, w)=p(w, w)=1)$, iff $a \geq \frac{1}{1-\delta}$ and $b \leq-\frac{1}{\delta}$,

(iii) there is a deterministic cyclical $\operatorname{EPCF}(p(x, y)=p(y, w)=p(z, w)=$ $p(w, x)=1)$, iff $a \geq 1+\delta$ and $b \geq-\frac{1}{\delta}$.

\section{Stochastic and symmetric EPCFs}

(i) there is a stochastic (symmetric) EPCF with its unique limit at $w$ $\left(p(x, y)=p(x, z)=\frac{1}{2}, p(y, w)=p(z, w)=p(w, w)=1\right)$, iff $b \leq-a-\frac{2}{\delta}$,

(ii) there is a stochastic (symmetric) cyclical EPCF $(p(x, y)=p(x, z)=$ $p, p(y, w)=p(z, w)=p(w, x)=1)$, iff $-a-\frac{2}{\delta} \leq b \leq\left(\frac{1+\delta+\delta^{2}}{\delta+\delta^{2}}\right) a-\frac{2}{\delta}$. Moreover, if $a \geq 1+\delta($ resp. $a<1+\delta)$, then $p=\frac{1}{2}\left(\right.$ resp. $\left.p<\frac{1}{2}\right)$.

Insert Figure 2 approximately here.

Observation 2 outlines a rich array of possible outcomes. In what follows we discuss the outcomes thoroughly. As a pictorial summary, Figure 2 depicts the various regions - in the space of parameters describing the defection and "sucker" payoffs - for which different outcomes obtain. For simplicity, the diagram has only been constructed for the limit case as $\delta \rightarrow 1$.

Now for a verbal account. Begin with deterministic equilibrium processes. Case 1(a) permits cooperation to be sustained as the unique limit of a deterministic EPCF as long as (and only if) $a$ is not too large. Although this finding is not unintuitive, it provides a different perspective on the relationship between our solution concept and the largest consistent set (LCS) in [11]. It is easy to see that the LCS is simply the singleton $\{x\}$ no matter what values $a$ and $b$ take (provided, of course, that $a>1$ and $b<0)$. However, no EPCF supports $x$ if $a$ is too large even when $\delta$ is close to unity.

This observation does not contradict Theorem 4.3, in which a deterministic EPCF with absorbing limit was shown to lie within the LCS. The 
point is that once $a$ is large enough, such EPCFs fail to exist. Cycles occur (as Case 1(c) illustrates), but Theorem 4.3 is silent on cyclical EPCFs. ${ }^{26}$

Another seeming contradiction to Theorem 4.3 is Case 1(b), which asserts that a deterministic EPCF may support $w$ as a unique absorbing limit. Notice, however, that the existence of such a scheme is conditional on $\delta$ not being too large, whereas Theorem 4.3 only applies for discount factors sufficiently close to unity.

Taken together, Cases 1(a) and 1(b) reveal two things. For discount factors large enough, if a deterministic EPCF with unique absorbing limit exists, then it can only sustain cooperation rather than defection. If, in addition, $a$ is too large then the existence of such EPCFs is jeopardized: Case 1(c) shows that in such cases one typically cycles between cooperation and defection.

The remarks so far pertain to deterministic schemes. Stochastic EPCFs tell a different story. Case 2(a) tells us that in contrast to the deterministic case, it is possible (even when $\delta \simeq 1$ ) to construct stochastic schemes with unique absorbing limit at mutual defection. The condition for this to happen can be interpreted in the form of a low enough value of $b$, the so-called "sucker payoff". That $b$ matters is not surprising, as this payoff (induced by the other agent's departure from cooperation) is what creates the "meta-coordination failure" discussed in the context of Example 11.

Finally, Case 2(b) identifies (necessary and sufficient) conditions for the presence of stochastic EPCFs that exhibit cycles. Notice that if $a$ is not too large then the cooperative outcome must exhibit some inertia along this cycle $(p(x, x)>0)$.

It may be worth pointing out that the conditions identified in cases 1(c) and 2(b) apply for the entire range of values for $a$ and $b$. In particular, we can use these conditions to conclude that no (symmetric) coordination game can exhibit a cycle.

The preceding discussion should make clear that cardinalities do matter in determining the sort of EPCF that drives any given Prisoners' Dilemma. To emphasize this and to focus on the leading case in which $\delta$ is close to unity, we end this section (and the paper) with three examples.

Example 12-1. (Prisoners' Dilemma 1): No EPCF supports the unique dominant strategy Nash equilibrium (and the unique coalition proof Nash

\footnotetext{
${ }^{26}$ The reader might think that cycles are efficient anyway when $a$ is large enough. But this is not generally true, as no restriction is placed on the absolute size of $b$.
} 
equilibrium) as its absorbing state, but cooperation can be supported as the unique absorbing state of a deterministic EPCF for $\delta$ close to unity. ${ }^{27}$

\begin{tabular}{|c|c|c|}
\hline & $L$ & $R$ \\
\hline$T$ & 1,1 & $-\frac{1}{2}, \frac{3}{2}$ \\
\hline$B$ & $\frac{3}{2},-\frac{1}{2}$ & 0,0 \\
\hline
\end{tabular}

ExAmple 12-2. (Prisoners' Dilemma 2): No EPCF supports cooperation, but the Nash equilibrium can be supported as the unique absorbing state of a stochastic EPCF for $\delta$ close to unity.

\begin{tabular}{|c|c|c|}
\hline & $L$ & $R$ \\
\hline$T$ & 1,1 & $-6,3$ \\
\hline$B$ & $3,-6$ & 0,0 \\
\hline
\end{tabular}

Example 12-3. (Prisoners' Dilemma 3): Both cooperation and noncooperation states may be supported as the unique absorbing state of EPCFs (deterministic and stochastic, respectively) for $\delta$ close to unity.

\begin{tabular}{|c|c|c|}
\hline & $L$ & $R$ \\
\hline$T$ & 1,1 & $-4, \frac{3}{2}$ \\
\hline$B$ & $\frac{3}{2},-4$ & 0,0 \\
\hline
\end{tabular}

\section{APPENDIX A}

\section{Remarks on Efficient Moves}

We make some brief remarks on the notion of efficient moves in the definition of an EPCF. As observed in the main text, one might weaken the definition of an EPCF to allow for all profitable moves, not just the efficient ones. Call such an EPCF a weak EPCF.

1. The existence of weak EPCFs is obviously not an issue, because an EPCF is clearly a weak EPCF.

\footnotetext{
${ }^{27}$ This game also has a stochastic cyclical EPCF with $p(x, x) \in(0,1)$.
} 
2. Weak EPCFs might lead to outcomes that appear unreasonable. Consider the following example with two players - 1 and $2-$ and three states, $x, y$ and $z$. Payoffs are as follows:

\begin{tabular}{|c|c|c|c|}
\hline$u_{i}\left(x^{\prime}\right)$ & $x$ & $y$ & $z$ \\
\hline 1 & 0 & 1 & 100 \\
\hline 2 & 1 & 0 & 100 \\
\hline
\end{tabular}

Suppose further that individual 1 can induce $y$ and $z$ from $x$, while 2 can induce $x$ and $z$ from $y$, and that no other coalition/state combination permits nontrivial moves.

Now it is easy to construct a weak EPCF (for all discount factors, in fact) in which, starting from either $x$ or $y$, the system endlessly oscillates between $x$ and $y$, even though either player could induce $z$ and make both players much better off. An EPCF would negate this possibility by permitting indeed, demanding - that each player make an efficient move.

3. At the same time, it is worth noting that our core characterization theorems may be strengthened by taking note of the distinction between EPCFs and weak EPCFs. This is true in the following sense. In Theorem 4.1, a (strong) core outcome is "implemented" by an EPCF (satisfying the efficient moves principle). At the same time, a cursory glance at the proof of Theorem 4.2 will reveal that every weak deterministic EPCF with unique absorbing limit must pick out a (weak) core allocation. That is, Theorem 4.2 applies to the broader class of weak EPCFs.

4. It hardly needs to be mentioned that the "efficient moves" requirement need not lead to efficiency overall, for exactly the same reason that Nash equilibria need not be Pareto optimal. For instance, Observation 2 tells us that an EPCF may lead to mutual defection as its unique absorbing limit in the case of the Prisoners' Dilemma.

5. However, there is an important sense in which our equilibrium concept fails to capture certain aspects of "efficient moves". We have proceeded entirely in the spirit of dynamic games, in which the one-shot deviation principle is applied: players take not only the strategies of other players as given, they take as given their own strategies in the future. By the well known principle of Blackwell that "unimprovability implies optimality" in discounted situations, there is seemingly no loss of generality in doing this. ${ }^{1}$

\footnotetext{
${ }^{1}$ The equivalence between the unimprovability of a single-step move, and optimality, lies at the heart of modern theories of repeated games (see, for example, [1] or [15]).
} 
But the principle fails when "players" are coalitions (and also if players have vector-valued objectives). To create a profitable deviation for the coalition as a whole (or for every component of the vector payoff function), several moves may be needed. Indeed, this is behind the efficiency failure in Example 9. There, the coalition $\{1,2\}$ can engineer, if it so wishes, a move from one of the inefficient absorbing states $a$ or $c$. However, a move from $a$ only ends at $c$, and vice versa, so that both players cannot find it simultaneously worthwhile to participate in the proposed move. At the same time, if players $\{1,2\}$ were to simultaneously deviate at both $a$ and $c$, the "double deviation" would indeed be worthwhile.

This raises a conceptual issue. The principle of one-step deviations is built into our solution concept: individuals and coalitions at different dates are regarded as different individuals and coalitions. Therefore coalitions are as involved (in this conceptualization) in a game against themselves as against other coalitions. It is unclear whether this formulation should be dropped (compare this, for instance, with the literature on changing preferences, e.g., [25] and [32]). We tentatively retain it, despite the disturbing feature of Example 9.

6. Finally — while accepting the efficient moves principle - one might question the particular formulation adopted in our definition. For instance, one could rule out an efficient move for $S$ (as described by us) if there is some strict subset of $S$, say $T$, which can generate another change that makes its members still better off relative to the payoff under the efficient move (by $S$ ). In this case one might want to assign probability zero to the move by $S$ (and positive probability to the move by $T$ ). However, this refinement raises other issues. One interpretation of the probabilistic nature of a move is that Nature chooses a coalition randomly and permits it to enjoy a profitable deviation. In that case, the subset $T$ might be bound by the decisions of the entire coalition $S$. On the other hand, if this interpretation is rejected, then other problems arise. For instance, why restrict the search for better moves to subsets of $S$ and not other sets $T$ which share a common intersection with $S$ (where the intersecting members are allowed to go with the coalition that has the better move)? But this further refinement leads to possible circularities, rendering a conceptually satisfactory definition impossible. At the same time, it should be noted that such potential circularities in defining efficient moves - which we avoid by assumption - do not in any way preclude the study of cycles over time, which are allowed for in the definition. 


\section{APPENDIX B}

\section{Proofs Omitted in the Main Text}

Proof of Observation 1. The value functions of this game can be rewritten as follows:

$$
v_{i}\left(x^{\prime}, p\right)=u_{i}\left(x^{\prime}\right)+\delta\left\{p\left(x^{\prime}, y^{\prime}\right) v_{i}\left(y^{\prime}, p\right)+\left(1-p\left(x^{\prime}, y^{\prime}\right)\right) v_{i}\left(x^{\prime}, p\right)\right\},
$$

or

$$
\left(1-\delta+\delta p\left(x^{\prime}, y^{\prime}\right)\right) v_{i}\left(x^{\prime}, p\right)-\delta p\left(x^{\prime}, y^{\prime}\right) v_{i}\left(y^{\prime}, p\right)=u_{i}\left(x^{\prime}\right),
$$

for any $i \in N$, and any $\left(x^{\prime}, y^{\prime}\right) \in\{(x, y),(y, z),(z, x)\}$. The incentive to move from $x^{\prime}$ to $y^{\prime}$ is captured as follows:

$$
v_{i}\left(y^{\prime}, p\right)-v_{i}\left(x^{\prime}, p\right)=\frac{(1-\delta) v_{i}\left(y^{\prime}, p\right)}{1-\delta+\delta p\left(x^{\prime}, y^{\prime}\right)}-\frac{u_{i}\left(x^{\prime}\right)}{1-\delta+\delta p\left(x^{\prime}, y^{\prime}\right)}
$$

First, we find a symmetric equilibrium. Since the game is symmetric, we can describe a symmetric equilibrium in the following way:

$$
\left(\begin{array}{ccc}
1-\delta+\delta p & 0 & -\delta p \\
-\delta p & 1-\delta+\delta p & 0 \\
0 & -\delta p & 1-\delta+\delta p
\end{array}\right)\left(\begin{array}{c}
v^{H} \\
v^{M} \\
v^{L}
\end{array}\right)=\left(\begin{array}{l}
1 \\
a \\
0
\end{array}\right)
$$

where

$$
\left(\begin{array}{c}
v^{H} \\
v^{M} \\
v^{L}
\end{array}\right)=\left(\begin{array}{l}
v_{1}(x, p) \\
v_{1}(z, p) \\
v_{1}(y, p)
\end{array}\right)=\left(\begin{array}{c}
v_{2}(y, p) \\
v_{2}(x, p) \\
v_{2}(z, p)
\end{array}\right)=\left(\begin{array}{l}
v_{3}(z, p) \\
v_{3}(y, p) \\
v_{3}(x, p)
\end{array}\right)
$$

By solving this equation we see that

$$
\begin{aligned}
v^{H} & =\frac{1}{D}\left\{(1-\delta+\delta p)^{2}+a(\delta p)^{2}\right\} \\
v^{M} & =\frac{1}{D}\left\{a(1-\delta+\delta p)^{2}+\delta p(1-\delta+\delta p)\right\} \\
v^{L} & =\frac{1}{D}\left\{(\delta p)^{2}+a \delta p(1-\delta+\delta p)\right\},
\end{aligned}
$$

where $D=(1-\delta+\delta p)^{3}-(\delta p)^{3}>0$, and $p$ denotes the probability of moving to the next state.

Note first that $v^{M}-v^{L}=(1-\delta)\{\delta p+a(1-\delta+\delta p)\}>0$. Thus, a player who is currently getting 0 surely joins a coalitional move. The question is 
whether a player who is currently getting $a$ would also do so. This can be checked by comparing $v^{H}$ and $v^{M}$ :

$$
\begin{aligned}
v^{H}-v^{M} & =\frac{1}{D}\left\{(1-\delta+\delta p)(1-\delta)+a(\delta p)^{2}-a(1-\delta+\delta p)^{2}\right. \\
& =\frac{1}{D}(1-\delta)\{(1-\delta+\delta p)-a(1-\delta+2 \delta p)\}
\end{aligned}
$$

Hence, we obtain

$$
v^{H} \geq(<) v^{M} \Longleftrightarrow a \leq(>) \frac{1-\delta+\delta p}{1-\delta+2 \delta p} .
$$

Note that $p=1$ if $v^{H}>v^{M}$, and $p \in(0,1)$ can occur only if $v^{H}=v^{M}$. Thus, when $a<\frac{1}{1+\delta}, v^{H}>v^{M}$ holds for any $p$, and we must conclude that $p=1$. Similarly, when $a=\frac{1}{1+\delta}$, the only possibility is, again, $p=1$.

When $a>\frac{1}{1+\delta}, p$ can no longer be 1 , since $p=1$ implies $v^{H}<v^{M}$. Since $a<1$, neither can it be that $p=0\left(v^{H}>v^{M}\right)$. Thus, the only possibility left is the case where $v^{H}=v^{M}$ so that $p \in(0,1)$ holds. Hence, when $a>\frac{1}{1+\delta}, p=\frac{(1-a)(1-\delta)}{\delta(2 a-1)}$ is the unique symmetric EPCF.

The rest of the proof shows that no other EPCF exists. To do that, we need to investigate a few possibilities. We first show that this game does not possess an EPCF that has an absorbing state for any $\delta$ and $a$. Suppose, to the contrary, that $p(x, x)=1$ (i.e., $x$ is an absorbing state). Then, $v_{i}(x, p)=\frac{u_{i}(x)}{1-\delta}$. Given this, the incentives for players 1 and 2 to move from $z$ to $x$ can be described by the difference between $v_{i}(x, p)$ and $v_{i}(z, p)$ :

$$
\begin{aligned}
v_{1}(x, p)-v_{1}(z, p) & =\left[\frac{(1-\delta) \frac{1}{1-\delta}}{(1-\delta+\delta p(z, x))}+\frac{a}{(1-\delta+\delta p(z, x))}\right] \\
& =\frac{1-a}{(1-\delta+\delta p(z, x))}>0
\end{aligned}
$$

and

$$
\begin{aligned}
v_{2}(x, p)-v_{2}(z, p) & =\frac{(1-\delta) \frac{a}{1-\delta}}{(1-\delta+\delta p(z, x))} \\
& =\frac{a}{(1-\delta+\delta p(z, x))}>0 .
\end{aligned}
$$

Hence, given that $x$ is an absorbing state, it must be that $p(z, x)=1$. Consequently, the discounted payoffs at $z$ are

$$
v_{i}(z, p)=u_{i}(z)+\frac{\delta}{1-\delta} u_{i}(x) .
$$


Now, we can check the incentives for players 1 and 3 to move from $y$ to $z$ :

$$
v_{1}(z, p)-v_{1}(y, p)=\frac{(1-\delta) v_{1}(z, p)}{1-\delta+\delta p(y, z)}>0,
$$

and

$$
\begin{aligned}
v_{3}(z, p)-v_{3}(y, p) & =\frac{(1-\delta) v_{2}(z, p)}{1-\delta+\delta p(y, z)}-\frac{a}{1-\delta+\delta p(y, z)} \\
& =\frac{1-\delta-a}{1-\delta+\delta p(y, z)} .
\end{aligned}
$$

Suppose that $1-\delta<a$. Then it must be that $p(y, z)=0$. However, if this is so, $y$ is also an absorbing state, and by repeating the same argument as before, players 2 and 3 would move from $x$ with probability $1(p(x, y)=1)$. This is a contradiction. Next suppose that $1-\delta \geq a$. Then, by the argument above, we must have $p(y, z) \in(0,1]$. We check if there is any $p(y, z) \in(0,1]$ that can support $p(x, y)=0$. The discounted sum of payoffs at $y$ can be written as

$$
(1-\delta+\delta p(y, z)) v_{i}(y, p)=u_{i}(y)+\delta p(y, z) v_{i}(z, p) .
$$

Therefore, we obtain

$$
v_{i}(y, p)=\frac{1}{1-\delta+\delta p(y, z)}\left[u_{i}(y)+\delta p(y, z)\left\{u_{i}(z)+\frac{u_{i}(x)}{1-\delta}\right\}\right]
$$

Now, at state $x$, we check if $p(x, y)=0$ is supportable. Player $i$ 's incentive to move from $x$ to $y$ is given by

$$
\begin{aligned}
v_{i}(y, p)-v_{i}(x, p) & =\frac{u_{i}(y)+\delta p(y, z) u_{i}(z)+\delta p(y, z) \frac{u_{i}(x)}{1-\delta}}{1-\delta+\delta p(y, z)}-\frac{u_{i}(x)}{1-\delta} \\
& =\frac{u_{i}(y)+\delta p(y, z) u_{i}(z)-(1+\delta) u_{i}(x)}{1-\delta+\delta p(y, z)} .
\end{aligned}
$$

Obviously, $v_{3}(y, p)-v_{3}(x, p)>0$. For player 2 , we can utilize $1-\delta \geq a$ to show $v_{2}(y, p)-v_{2}(x, p)>0$.

$$
\begin{aligned}
v_{2}(y, p)-v_{2}(x, p) & =\frac{1}{1-\delta+\delta p(y, z)}[1-(1+\delta) a] \\
& \geq \frac{1}{1-\delta+\delta p(y, z)}[1-(1+\delta)(1-\delta)]>0
\end{aligned}
$$


This is a contradiction to $p(x, y)=0$. As a result, we conclude that for any $a$ and $\delta$, this game does not possess an absorbing EPCF.

So far, we know that $p\left(x^{\prime}, y^{\prime}\right) \in(0,1]$ must hold for any $\left(x^{\prime}, y^{\prime}\right) \in$ $\{(x, y),(y, z),(z, x)\}$. Now, we will show that $p\left(x^{\prime}, y^{\prime}\right) \in(0,1)$ for any $\left(x^{\prime}, y^{\prime}\right) \in\{(x, y),(y, z),(z, x)\}$ unless $p(x, y)=p(y, z)=p(z, x)=1$. Two sub-cases need to be investigated. The first one is the case with only one deterministic move. Without loss of generality, we assume $p(y, z)=1$. Since $p(x, y) \in(0,1)$, we have

$$
v_{2}(y, p)=1+\delta v_{2}(z, p)=\frac{a}{1-\delta}
$$

and

$$
v_{2}(z, p)=\frac{\delta p(z, x)}{1-\delta+\delta p(z, x)} v_{2}(x, p)=\frac{\delta p(z, x)}{1-\delta+\delta p(z, x)} \frac{a}{1-\delta}
$$

These two equations together imply

$$
1+\frac{\delta^{2} p(z, x)}{1-\delta+\delta p(z, x)} \frac{a}{1-\delta}=\frac{a}{1-\delta},
$$

which is equivalent to

$$
p(z, x)=\frac{a-(1-\delta)}{\delta(1-a)} .
$$

Since $p(z, x) \leq 1$, we must have $\frac{1}{1+\delta} \geq a$. Now, consider player 1 . Since $p(z, x) \in(0,1)$, we have $v_{1}(z, p)=v_{1}(x, p)=\frac{a}{1-\delta}$. Because we have that $(1-\delta+\delta p(x, y)) v_{1}(x, p)=1+\delta p(x, y) v_{1}(y, p)$ and $v_{1}(y, p)=\delta v_{1}(z, p)$, it follows that

$$
\left(1-\delta+\delta p(x, y)-\delta^{2} p(x, y)\right) \frac{a}{1-\delta}=1
$$

or

$$
p(x, y)=\frac{1-a}{a} .
$$

Since $p(x, y) \leq 1$, we have $\frac{1}{2} \leq a$. Together with the previous result, this implies $\frac{1}{2} \leq a \leq \frac{1}{1+\delta}$. But this contradicts the fact that $\delta<1$.

Next, we consider the remaining subcase in which two moves are deterministic. Suppose, without loss of generality, that $p(x, y) \in(0,1)$ and $p(y, z)=p(z, x)=1$. In this case, player 2 has to be indifferent between $x$ and $y$. Thus, $v_{2}(x, p)=v_{2}(y, p)=\frac{a}{1-\delta}$. Since $v_{2}(y, p)=1+\delta \cdot 0+\delta^{2} v_{2}(x, p)$, 
we have $\left(1-\delta^{2}\right) v_{2}(x, p)=1$. By equation these two equations, we obtain $a=\frac{1}{1+\delta}$. Now, we focus on player 3's incentive to move from $y$ to $z$. We have:

$$
\begin{aligned}
& v_{3}(x, p)=\frac{\delta p(x, y)}{1-\delta+\delta p(x, y)} v_{3}(y, p), \\
& v_{3}(y, p)=a+\delta+\delta^{2} v_{3}(x, p) \\
& v_{3}(z, p)=1+\delta v_{3}(x, p) .
\end{aligned}
$$

From the first two equations, we obtain:

$$
v_{3}(x, p)=\frac{\delta p(x, y)}{1-\delta+\delta p(x, y)}\left(a+\delta+\delta^{2} v_{3}(x, p)\right) .
$$

Substituting $a=\frac{1}{1+\delta}$ into this equation, we obtain,

$$
(1-\delta)(1+\delta(1+\delta) p(x, y)) v_{3}(x, p)=\delta p(x, y) \frac{1+\delta+\delta^{2}}{1+\delta} .
$$

Thus, we have a formula for $v_{3}(x, p)$,

$$
v_{3}(x, p)=\frac{\delta\left(1+\delta+\delta^{2}\right) p(x, y)}{(1-\delta)(1+\delta)(1+\delta(1+\delta) p(x, y))} .
$$

Now, to check the incentive to move from $y$ to $z$, we calculate $v_{3}(z, p)-$ $v_{3}(y, p)$. This is given by

$$
\begin{aligned}
v_{3}(z, p)-v_{3}(y, p) & =1-a-\delta+\delta(1-\delta) v_{3}(x, p) \\
& =-\frac{\delta^{2}}{1+\delta}+\frac{\delta^{2}\left(1+\delta+\delta^{2}\right) p(x, y)}{(1+\delta)\left(1+\left(\delta+\delta^{2}\right) p(x, y)\right)} \\
& \leq-\frac{\delta^{2}}{1+\delta}+\frac{\delta^{2}\left(1+\delta+\delta^{2}\right)}{(1+\delta)\left(1+\delta+\delta^{2}\right)}=0 .
\end{aligned}
$$

Equality holds only when $p(x, y)=1$. But this contradicts our supposition that $p(x, y) \in(0,1)$. Therefore, we may conclude that $p\left(x^{\prime}, y^{\prime}\right)=1$ cannot hold for any $\left(x^{\prime}, y^{\prime}\right) \in\{(x, y),(y, z),(z, x)\}$.

Finally, we check if there is an asymmetric EPCF when $p\left(x^{\prime}, y^{\prime}\right) \in(0,1)$ for any $\left(x^{\prime}, y^{\prime}\right) \in\{(x, y),(y, z),(z, x)\}$. Since $p\left(x^{\prime}, y^{\prime}\right)$ is strictly mixed, there must be player $i$ in $S$ with $y^{\prime} \in F_{S}\left(x^{\prime}\right)$ who is indifferent between staying at $x^{\prime}$ and moving to $y^{\prime}$ : that is,

$$
v_{i}\left(y^{\prime}, p\right)-v_{i}\left(x^{\prime}, p\right)=\frac{(1-\delta) v_{i}\left(y^{\prime}, p\right)}{1-\delta+\delta p\left(x^{\prime}, y^{\prime}\right)}-\frac{u_{i}\left(x^{\prime}\right)}{1-\delta+\delta p\left(x^{\prime}, y^{\prime}\right)}=0,
$$


so that

$$
v_{i}\left(x^{\prime}, p\right)=v_{i}\left(y^{\prime}, p\right)=\frac{u_{i}\left(x^{\prime}\right)}{1-\delta} .
$$

Note that the indifferent players are players 2,3 , and 1 for $\left(x^{\prime}, y^{\prime}\right)=(x, y)$, $(y, z)$, and $(z, x)$, respectively. Since everything is symmetric, we focus on the case of $\left(x^{\prime}, y^{\prime}\right)=(x, y)$. In this case, player 2 is indifferent between $x$ and $y$. Since $v_{2}(z, p)=0+\delta\left(p(z, x) v_{2}(x, p)+(1-p(z, x)) v_{2}(z, p)\right)$, we obtain

$$
v_{2}(z, p)=\frac{\delta p(z, x)}{1-\delta+\delta p(z, x)} v_{2}(x, p) .
$$

Similarly, we know $v_{2}(y, p)=1+\delta\left(p(y, z) v_{2}(z, p)+(1-p(y, z)) v_{2}(y, p)\right)$. Substituting the above equation and $v_{2}(x, p)=v_{2}(y, p)=\frac{a}{1-\delta}$ into this equation, we obtain,

$$
\frac{a}{1-\delta}=1+\frac{a}{1-\delta}\left\{\frac{\delta p(y, z) \delta p(z, x)}{1-\delta+\delta p(z, x)}+\delta(1-p(y, z))\right\}
$$

This is equivalent to

$$
\frac{1-\delta+\delta p(z, x)}{1-\delta+\delta p(z, x)+\delta p(y, z)}=a
$$

Repeating the same argument for players 1 and 3 , we obtain

$$
\begin{aligned}
\frac{1-\delta+\delta p(z, x)}{1-\delta+\delta p(z, x)+\delta p(y, z)} & =\frac{1-\delta+\delta p(x, y)}{1-\delta+\delta p(x, y)+\delta p(z, x)} \\
& =\frac{1-\delta+\delta p(y, z)}{1-\delta+\delta p(y, z)+\delta p(x, y)} \\
& =a .
\end{aligned}
$$

Suppose that $\delta p(y, z)<\delta p(z, x)$. Then, we have $\delta p(z, x)<\delta p(x, y)$ and $\delta p(x, y)<\delta p(y, z)$. However, these two inequalities together imply $\delta p(z, x)<\delta p(y, z)$, a contradiction. Thus, asymmetric probabilities within an open interval $(0,1)$ cannot survive. Note that if $p(x, y)=p(y, z)=$ $p(z, x)$, then we indeed obtain $p(x, y)=\frac{(1-a)(1-\delta)}{\delta(2 a-1)}$.

Proof of Observation 2. First, note that in any EPCF, $p(y, w)=$ $p(z, w)=1$, since $y$ and $z$ give players 1 and 2 the minimum payoff, respectively (this implies $p(y, y)=p(z, z)=0$, and to move to $w$ is the only incentive compatible path). We start with deterministic EPCFs. 
Case 1.a. Deterministic EPCFs with unique absorbing state $x$. From Theorem 4.3, we know that $x$ can be the only absorbing state of a deterministic EPCF when $\delta$ is close to one, so we first find a deterministic EPCF with its absorbing state at $x$.

For this to happen, we must have in particular that $p(w, x)=1$. This in turn requires that (i) $v_{i}(w, p) \leq v_{i}(x, p)$. Moreover, since $x$ is an absorbing state $(p(x, x)=1)$, we need (ii) $v_{1}(x, p) \geq v_{1}(y, p)$ (since the EPCF is symmetric, we can focus on player 1$)$. First, let us consider (i). Since $p(x, x)=1, v_{i}(x, p)=\frac{1}{1-\delta}>0$, and $v_{i}(w, p)=0+\delta v_{i}(x, p)$. Obviously, (i) is satisfied with a strict inequality. Thus, $p(w, x)=1$ is incentive compatible. Second, (ii) is investigated. Since the EPCF is fully specified by the previous analysis, it is easy to see the value of $v_{1}(y, p): v_{1}(y, p)=$ $a+\delta v_{1}(w, p)=a+\delta^{2} v_{1}(x, p)$. Thus, we have

$$
\begin{aligned}
v_{1}(x, p)-v_{1}(y, p) & =\left(1-\delta^{2}\right) v_{1}(x, p)-a \\
& =\left(1-\delta^{2}\right) \frac{1}{1-\delta}-a \\
& =1+\delta-a \geq 0 .
\end{aligned}
$$

Thus, if (and only if) $a \leq 1+\delta$, we can support $x$ as the unique absorbing state of a deterministic EPCF.

Case 1.b. Deterministic EPCFs with unique absorbing state $w$. We analyze a PCF with $p(x, y)=p(y, w)=p(z, w)=p(w, w)=1$. [Since it is deterministic, the EPCF must treat moves to $y$ and $z$ asymmetrically. The only other case is the mirror image of this, to be analyzed in exactly the same way.]

Since $p(y, w)=p(z, w)=1$ always follows, we only need to focus on (i) $p(x, y)=1$ and (ii) $p(w, w)=1$ (or $p(w, x)=0$ ). First, we analyze (i): In order to give player 1 an incentive to move from $x$, we need $a+\delta v_{1}(w, p) \geq$ $v_{1}(x, p)$. Note that $v_{1}(x, p)=1+\delta a+\delta^{2} v_{1}(w, p)$ and $v_{1}(w, p)=0$. Thus, what we need is $a \geq 1+\delta a$ or $a \geq \frac{1}{1-\delta}$. Second, we check (ii): It is sufficient to show that player 2 (who suffers more by moving to $x$ ) does not agree with moving to $x$. Thus, we need $0=v_{2}(w, p) \geq v_{2}(x, p)=$ $1+\delta b+\delta^{2} v_{2}(w, p)=1+\delta b$. Therefore, if (and only if) (i) $a \geq \frac{1}{1-\delta}$ and (ii) $b \leq-\frac{1}{\delta}$ are satisfied, then $w$ can be supported as the unique absorbing state of a deterministic EPCF.

Case 1.c. Deterministic cyclical EPCF. We analyze a PCF with $p(x, y)=$ $p(y, w)=p(w, x)=1$. We need two conditions: (i) $a+\delta v_{1}(w, p) \geq v_{1}(x, p)$, and (ii) $v_{2}(x, p) \geq v_{2}(w, p)$ (since player 2 suffers more by moving to $x$ ). 
However, we know

$$
\begin{gathered}
v_{i}(w, p)=\delta v_{i}(x, p) \text { for } i=1,2, \\
v_{1}(x, p)=\left(1+\delta a+\delta^{2} 0\right)+\delta^{3}\left(1+\delta a+\delta^{2} 0\right)+\ldots=\frac{1+\delta a}{1-\delta^{3}},
\end{gathered}
$$

and

$$
v_{2}(x, p)=\left(1+\delta b+\delta^{2} 0\right)+\delta^{3}\left(1+\delta b+\delta^{2} 0\right)+\ldots=\frac{1+\delta b}{1-\delta^{3}}
$$

Thus, condition (i) becomes

$$
a \geq\left(1-\delta^{2}\right) v_{1}(x, p)=\left(1-\delta^{2}\right) \frac{1+\delta a}{1-\delta^{3}}=\frac{(1+\delta)(1+\delta a)}{1+\delta+\delta^{2}} .
$$

This is equivalent to $a \geq 1+\delta$. Condition (ii) boils down to $v_{2}(x, p) \geq 0$, which is equivalent to $b \geq-\frac{1}{\delta}$. Hence, if (i) $a \geq 1+\delta$ and (ii) $b \geq-\frac{1}{\delta}$ are satisfied, then this cyclical deterministic EPCF is supportable.

Case 2.a. Stochastic symmetric EPCFs with an absorbing state $w$. We can set up value functions in a similar way as we did in Example 8. We focus on player 1 without loss of generality (symmetry):

$$
\begin{aligned}
v_{1}(x, p) & =1+\frac{\delta}{2}\left(v_{1}(y, p)+v_{1}(z, p)\right), \\
v_{1}(y, p) & =a+\delta v_{1}(w, p), \\
v_{1}(z, p) & =b+\delta v_{1}(w, p), \\
v_{1}(w, p) & =0 .
\end{aligned}
$$

By substituting $v_{1}(w, p)=0$ to others, we obtain

$$
\begin{aligned}
& v_{1}(x, p)=1+\frac{\delta}{2}(a+b), \\
& v_{1}(y, p)=a, \\
& v_{1}(z, p)=b .
\end{aligned}
$$

Since $a<0$ and $b<0, p(y, w)=p(z, w)=1$ are incentive compatible. Moreover, $p(w, w)=1$ can be supported if $v_{1}(x, p) \leq 0$, or $a+b \leq-\frac{2}{\delta}$. Now, the question is if we can support $p(x, y)=\frac{1}{2}$. The answer is yes, if $v_{1}(y, p) \geq v_{1}(x, p)$, or $a \geq \frac{2}{2-\delta}+\frac{\delta}{2-\delta} b$. Thus, if (i) $a+b \leq-\frac{2}{\delta}$, and (ii) $a \geq \frac{2}{2-\delta}+\frac{\delta}{2-\delta} b$, then the above PCF is indeed an EPCF. ${ }^{1}$ Since condition

\footnotetext{
${ }^{1}$ We can also consider a PCF with $p(y, w)=p(z, w)=p(w, w)=1, p(x, y)=p(x, z)=$ $p$, and $p(x, w)=1-2 p$ for $p \in\left(0, \frac{1}{2}\right)$. It is easy to see that whenever there is such an $\mathrm{EPCF}$, we also have an EPCF described in the main text.
} 
(ii) is satisfied trivially ( $a$ is the highest payoff of all), condition (i) is the only one that needs to be satisfied. That is, if $a+b \leq-\frac{2}{\delta}$, then $w$ can be supported as unique absorbing state of a symmetric stochastic EPCF.

Case 2.b. Stochastic symmetric cyclical EPCFs. We analyze a PCF with $p(x, y)=p(x, z)=p \leq \frac{1}{2}$, and $p(y, w)=p(x, w)=p(w, x)=1$. Again, the key conditions are (i) $v_{1}(y, p) \geq v_{1}(x, p)$, and (ii) $v_{1}(x, p) \geq v_{1}(w, p)$. The formula of $v_{1}(x, p)$ can be written as:

$$
\begin{aligned}
v_{1}(x, p) & =1+\delta\left\{p\left(v_{1}(y, p)+v_{1}(z, p)\right)+(1-2 p) v_{1}(x, p)\right\} \\
& =1+\delta p(a+b)+2 \delta^{2} p v_{1}(w, p)+\delta(1-2 p) v_{1}(x, p) \\
& =1+\delta p(a+b)+2 \delta^{3} p v_{1}(x, p)+\delta(1-2 p) v_{1}(x, p) .
\end{aligned}
$$

Thus, we have

$$
\left\{1-\delta+2 \delta p-2 \delta^{3} p\right\} v_{1}(x, p)=1+\delta p(a+b),
$$

or

$$
v_{1}(x, p)=\frac{1+\delta p(a+b)}{(1-\delta)(1+2 \delta p(1+\delta))} .
$$

Now, we are ready to check condition (i). We need $v_{1}(y, p)=a+\delta^{2} v_{1}(x, p) \geq$ $v_{1}(x, p)$, or $\frac{a}{1-\delta^{2}} \geq v_{1}(x, p)$. Thus, we need

$$
\frac{1+\delta p(a+b)}{(1-\delta)(1+2 \delta p(1+\delta))} \leq \frac{a}{(1-\delta)(1+\delta)}
$$

or

$$
1+\delta p a+\delta p b \leq \frac{(1+2 \delta p(1+\delta)) a}{1+\delta}=\frac{a}{1+\delta}+2 \delta p a .
$$

Hence, condition (i) boils down to

$$
b \leq\left\{\frac{1}{(1+\delta) \delta p}+1\right\} a-\frac{1}{\delta p} .
$$

Now, condition (ii). This is equivalent to $v_{1}(x, p) \geq 0$, or $a+b \geq-\frac{1}{\delta p}$. These two conditions together, we finally obtain,

$$
-a-\frac{1}{\delta p} \leq b \leq\left\{\frac{1}{(1+\delta) \delta p}+1\right\} a-\frac{1}{\delta p} .
$$

Recall $p \in\left(0, \frac{1}{2}\right]$. Thus, an stochastic EPCF with $p(x, x)=0\left(p=\frac{1}{2}\right)$ can be supported in the parameter range of $-a-\frac{2}{\delta} \leq b \leq\left(\frac{2+\delta+\delta^{2}}{\delta+\delta^{2}}\right) a-\frac{2}{\delta}$. 
The second inequality is almost always satisfied if $a>1$ (the prisoners' dilemma) and $\delta$ close to unity. The first inequality is more demanding and we need $-2 \leq a+b$ in the case of $\delta$ being close to unity.

What if $p$ is less than $\frac{1}{2}$ ? In this case, $p(x, x)>0$ holds. To have this situation, players 1 and 2 need to be indifferent between deviating from $x$ and staying at $x$. This implies the second inequality needs to hold as an equality. Thus, we need have

$$
b=\left\{\frac{1}{(1+\delta) \delta p}+1\right\} a-\frac{1}{\delta p},
$$

or

$$
p=\frac{1-\frac{a}{1+\delta}}{\delta(a-b)} .
$$

Thus, if $a \geq 2$, then there is no such $p>0$ for any $\delta<1$, and we can only have a stochastic EPCF with $p=\frac{1}{2}$. If $a<2$, then the above $p$ satisfies the first inequality as long as $\delta>a-1$, and $p<\frac{1}{2}$ can form a stochastic EPCF.

\section{REFERENCES}

1. D. Abreu, On the theory of infinitely repeated games with discounting, Econometrica 56 (1988), 383-96.

2. R. Aumann and R. Myerson, Endogenous formation of links between players and of coalitions: An application of the Shapley value, in "The Shapley Value: Essays in Honor of Lloyd Shapley" (A. Roth, Ed.), pp. 175-191, Cambridge Univ. Press, Cambridge, 1988.

3. S. Banerjee, H. Konishi, and T. Sönmez, Core in a simple coalition formation game, Soc. Choice Welfare 18 (2001), 135-153.

4. D. Bernheim, B. Peleg and M. Whinston, Coalition-proof Nash equilibria. I. Concepts, J. Econ. Theory 42 (1987), 1-12.

5. F. Bloch, Endogenous structures of associations in oligopolies, Rand J. Econ. 26 (1995), 537-556.

6. F. Bloch, Sequential Formation of Coalitions with Fixed Payoff Division, Games Econ. Behav. 14 (1996), 90-123.

7. F. Bloch, Coalition formation in games with spillovers, in "New Directions in the Economic Theory of the Environment" (C. Carraro and D. Siniscalco, Eds.), pp. 311-352, Cambridge Univ. Press, Cambridge, 1997.

8. A. Bogomolnaia and M. Jackson, The stability of hedonic coalition structures, Games Econ. Behav. 38 (2002), 201-230.

9. K. Chatterjee, B. Dutta, D. Ray, and K. Sengupta, A noncooperative theory of coalitional bargaining, Rev. Econ. Stud. 60 (1993), 463-477.

10. I-K. Cho, K. Jewell, and R. Vohra, A simple model of coalitional bidding, Econ. Theory 19 (2002), 435-457. 
11. M. Chwe, Farsighted coalitional stability, J. Econ. Theory 63 (1994), 299-325.

12. B. Dutta and D. Ray, A concept of egalitarianism Under participation constraints, Econometrica 57 (1989), 615-635.

13. B. Dutta and S. Mutuswami, Stable networks, J. Econ. Theory 76 (1997), 322-344.

14. B. Dutta, D. Ray, K. Sengupta and R. Vohra, A consistent bargaining set, J. Econ. Theory 49 (1989), 93-112.

15. D. Fudenberg and E. Maskin, The folk theorem in repeated games with discounting or with incomplete information, Econometrica 54 (1986), 533-54.

16. J. Green, The stability of Edgeworth's recontracting process, Econometrica 42 (1974), 21-34.

17. J. Greenberg, "The Theory of Social Situations," Cambridge Univ. Press, Cambridge, 1990.

18. J. Harsanyi and R. Selten, "A General Theory of Equilibrium Selection in Games," MIT Press, Cambridge, 1988.

19. S. Hart and M. Kurz, Endogenous formation of coalitions, Econometrica 51 (1983), 1047-1064.

20. M. Jackson and A. Wolinsky, A strategic model of social and economic networks, $J$. Econ. Theory $\mathbf{7 1}$ (1996), 44-74.

21. M. Kandori, G. Mailath, and R. Rob, Learning, mutation, and long run equilibria in games, Econometrica 61 (1993), 29-56.

22. R. Lagunoff and A. Matsui, Asynchronous choice in repeated coordination games, Econometrica 65 (1997), 1467-1477.

23. M. Mariotti, A model of agreements in strategic form games, J. Econ. Theory $\mathbf{7 4}$ (1997), 196-217.

24. A. Okada, A noncooperative coalitional bargaining game with random proposers, Games Econ. Behav. 16 (1996), 97-108.

25. E. Phelps and R. Pollack, On second-best national saving and game-equilibrium growth, Rev. Econ. Stud. 35 (1968), 185-199.

26. D. Ray, Credible coalitions and the core, Int. J. Game Theory 18 (1989), 185-187.

27. D. Ray and R. Vohra, Equilibrium binding agreements, J. Econ. Theory 73 (1997), 30-78.

28. D. Ray and R. Vohra, A theory of endogenous coalition structures, Games Econ. Behav. 26 (1999), 286-336.

29. R. Rosenthal, Cooperative games in effectiveness form, J. Econ. Theory 5 (1972), 88-101.

30. A. Sengupta and K. Sengupta, A property of the core, Games Econ. Behav. 12 (1996), 266-73.

31. P. Shenoy, On coalition formation: a game theoretic approach, Int. J. Game Theory 8 (1979), 133-164.

32. R. Strotz, Myopia and inconsistency in dynamic utility maximization, Rev. Econ. Stud. 23 (1958), 165-180.

33. R. Thrall and W. Lucas, $n$-person games in partition function form, Naval Research Logistics Quarterly 10 (1963), 281-298.

34. L. Xue, Coalitional stability under perfect foresight, Econ. Theory 11 (1998), 603627.

35. S-S. Yi, Stable coalition structures with externalities, Games Econ. Behav. 20 (1997), 201-237. 


\section{Figure Captions}

FIG. 1. An Inefficient Outcome.

Figure 1 is supplied as ineff.eps.

FIG. 2. Symmetric EPCFs in the Prisoners' Dilemma.

Figure 2 is supplied as obs2.eps. 